\title{
ON A NON-VANISHING CONJECTURE OF KAWAMATA AND THE CORE OF AN IDEAL
}

\author{
EERO HYRY AND KAREN E. SMITH
}

\begin{abstract}
We show, under suitable hypothesis which are sharp in a certain sense, that the core of an m-primary ideal in a regular local ring of dimension $d$ is equal to the adjoint (or multiplier) ideal of its $d$-th power. This generalizes the fundamental formula for the core of an integrally closed ideal in a two dimensional regular local ring due to Huneke and Swanson. We also find a generalization of this result to singular (non-regular) settings, which we show to be intimately related to the problem of finding non-zero sections of ample line bundles on projective varieties. In particular, we show that a graded analog of our formula for core would imply a remarkable conjecture of Kawamata predicting that every adjoint ample line bundle on a smooth variety admits a non-zero section.
\end{abstract}

\section{Contents}

1. Introduction

1.1. The motivating Geometry

1.2. From Geometrv to Algebra. 4

1.3. The Main Algebraic Results. 5

2. Aloebraic Preliminaries. 6

2.1. Integral Closure. Reductions, and the Core 6

2.2. Canonical sheaves and trace. 8

2.3. Duality 9

2.4. Rees Rings and Associated Graded Rings. 10

2.5. The Sancho de Salas Sequence. 11

2.6. The Graded Canonical Module. 12

3. The Main Technical Theorem 15

3.1. The filtration by Adioint-tvpe modules. 16

3.2. The canonical module $\omega_{G}$ in the dimension one case. 18

3.3. Reduction to the Associated Graded Ring 20

3.4. The Proof for $\omega_{\mathrm{a}} \quad 22$

4. Briancon-Skoda Tvpe results. 27

4.1. The Briancon-Skoda Theorem and related properties of adjoints. 27

4.2. The case where $A$ is not necessarilv Cohen-Macaulav. 30

5. The main local algebraic results 33

1991 Mathematics Subject Classification. Primary 13 ; Secondary 14 .

Key words and phrases. effective non-vanishing, adjoint, core, multiplier ideal, Shokurov's non-vanishing.

Research of Hyry supported by the National Academy of Finland, project number 48556.

Research of Smith partially supported by a US Fulbright fellowship and the National Science Foundation DMS 00-70722. Both authors are grateful for the hospitality of Jyväkylä University where the bulk of this work was carried out. 
5.1. Formulas for Core in the Cohen-Macaulav case. 33

5.2. Core in Dimension one. 36

5.3. Core and adioints. 36

5.4. Core and coefficient ideals. 38

5.5. Further Properties of Core, and Questions 38

6. Non-Vanishing Sections and the Core 39

6.1. A general criterion for non-vanishing. 40

6.2. An Adioint Computation. 43

6.3. The section ring. 45

6.4. Core and Graded Core in Graded Rings 47

$\begin{array}{ll}\text { References } & 49\end{array}$

\section{INTRODUCTION}

Let $I$ be an ideal in a commutative ring. By definition, the core of $I$ is the intersection of all sub-ideals having the same integral closure as $I$. Because the notion of integral closure is so fundamental, the core is a natural and interesting object. Originally defined by Rees and Sally in [37, the first substantial progress understanding core is due to Huneke and Swanson in [22], who proved that the core of an integrally closed $\mathfrak{m}$-primary ideal $I$ in a two dimensional regular local ring is equal to the adjoint (or multiplier) ideal of $I^{2}$. Since then, the algebraic properties of core have been thoroughly studied; see, for example, [6] and [7]. Our own interest in the core is motivated by seemingly unrelated geometric concerns: the core of a certain ideal governs whether or not an ample line bundle on a projective algebraic variety has a non-zero global section.

The purpose of this paper is two-fold. On the algebraic side, we find structure theorems for the core naturally generalizing the Huneke-Swanson results to the higher dimensional and singular case. On the geometric side, we show how a sufficiently good understanding of the core of a certain ideal in a very special kind of graded ring would settle a remarkable conjecture of Kawamata predicting that "adjoint" nef divisors are always effective. In particular, our higher dimensional singular version of the Huneke-Swanson formula for core can be viewed as a local analog of Kawamata's conjecture. Our work shows that commutative algebraists and algebraic geometers, working completely independently of each other and motivated by very different problems, both discovered and conjectured different facets of the same beautiful - and still largely buried - mathematical diamond.

It is perhaps no surprise that multiplier (adjoint) ideals arise in the search for non-vanishing theorems for nef line bundles. In recent years, multiplier ideals have found many rich applications to algebraic geometry, particularly to issues of effectiveness; see [8], 42]. Originally defined as ideals of holomorphic functions belonging to a certain weighted $L^{2}$-space, multiplier ideals can also be developed purely algebraically (see [35]) or algebro-geometrically (see 9] or 31]). Recently, multiplier ideals have been used to prove surprising new results in commutative algebra as well; see [10] and [11]. 
1.1. The motivating Geometry. Let $X$ be a complex smooth projective variety and let $L$ be an ample line bundle on $X$. Being ample, the line bundle

$$
L^{n}=\underbrace{L \otimes L \otimes \cdots \otimes L}_{n \text { times }}
$$

has many global sections for large enough $n$. On the other hand, $L$ itself need not have any sections at all. A fundamental unanswered question in algebraic geometry is this: what $n$ is large enough so that $L^{n}$ has even one non-trivial global section?

As stated, there is no general answer to this question; there is no uniform $n$ that works for all line bundles on a given variety $X$. So we must restrict attention to particular classes of bundles. For example, suppose that $L$ is an adjoint bundle, that is, that $L$ is of the form $K_{X} \otimes H$ where $H$ is some ample line bundle and $K_{X}$ is the canonical bundle of $X$. The Kodaira vanishing theorem implies that the higher cohomology groups of $L$ must all vanish, but as pointed out by Ambro in 1, not a single example is known in which the zeroth cohomology group also vanishes. In fact, even for numerically effective ("nef") line bundles $L$ of the form $K_{X} \otimes H$ where $H$ is ample, the celebrated theorem of Shokurov guarantees that $L^{n}$ has many sections for sufficiently large $n$ [40; again, in all known examples, $L$ itself already has a non-zero section.

In [25], Kawamata conjectured that every numerically effective line bundle $L$ adjoint to an ample line bundle must have a non-zero global section: that is, if $L$ is of the form $K_{X} \otimes H$ for some ample $H$, then $L^{n}$ has a non-zero section for every $n \geq 1$. More generally, Kawamata stated his effective non-vanishing conjecture in a singular, logarithmic version, indeed, under the same general hypothesis of Shokurov's non-vanishing theorem (or of the "Base Point Free Theorem") 25]:

Conjecture 1.1.1. Let D be any numerically effective ("nef") Cartier divisor on a normal projective variety $X$. If there exists an effective $\mathbb{R}$-divisor $B$ such that the pair $(X, B)$ is Kawamata log terminal ${ }^{1}$ and such that the $\mathbb{R}$-Cartier divisor $D-\left(K_{X}+B\right)$ is big and nef, then the line bundle $\mathcal{O}_{X}(D)$ has a non-zero global section.

This remarkably strong conjecture was first raised as a question by Ambro in 11. For curves it is trivially true and for surfaces it can be shown using a Riemann-Roch argument (25. Theorem 3.8]), but in higher dimensions it seems quite surprising. For example, Conjecture 1.1.1 predicts that the linear system of every nef divisor on a smooth Fano variety is non-empty; in particular, every Fano variety admits an effective anti-canonical divisor. For smooth varieties for which $K_{X}$ is trivial, Conjecture 1.1.1 asserts that every big and nef line bundle has a non-zero section; in particular, every ample line bundle on a Calabi-Yau manifold admits a non-trivial global section. At the opposite extreme, Conjecture 1.1.1 guarantees the existence of non-zero sections for the bi-canonical bundle on a smooth minimal model of general type.

\footnotetext{
${ }^{1}$ One can get interesting, but less technical statements by taking $B$ to be zero and $X$ to be Gorenstein: then the condition that $(X, B)$ is Kawamata log terminal amounts to $X$ having rational singularities. For the basic terminology of singularities of pairs used here, we recommend 29] or [30] as a general reference.
} 
1.2. From Geometry to Algebra. Kawamata has shown that in order to prove Conjecture 1.1.1 it suffices to prove the case where $D$ is ample 25. Theorem 2.2]. This opens up the possibility of using commutative algebra.

Given a pair $(X, D)$ consisting of a normal projective variety $X$ and an ample divisor $D$, consider the section ring

$$
S=\bigoplus_{n \in \mathbb{N}} H^{0}\left(X, \mathcal{O}_{X}(n D)\right),
$$

a normal, finitely generated graded ring whose associated projective scheme recovers $X$. As we describe in detail in Section 6, Kawamata's Conjecture leads naturally to an equivalent statement about the graded core of a certain submodule of the canonical module $\omega_{S}$ of $S$. This statement takes its simplest form in the case where $D=-K_{X}$ is the anti-canonical bundle on a smooth Fano variety. In this case, the section ring $S$ is Gorenstein and the line bundle $-K_{X}$ will have a non-zero section if the formula

$$
\operatorname{gradedcore}(I)=\operatorname{adj}\left(I^{d}\right), \quad \text { where } d=\operatorname{dim} S,
$$

holds for the integrally closed ideal $I=S_{\geq n}$ generated by elements of degrees at least $n$, for some (equivalently, every) fixed large $n$. Here the graded core of $I$ is the intersection of all homogeneous subideals whose integral closure is $I$, and $\operatorname{adj}\left(I^{d}\right)$ is the adjoint ideal (or multiplier ideal) of $I^{d}$. (The definition is recalled in Section [5.3).

For more general pairs $(X, D)$, the section ring $S$ will not be Gorenstein, so the usual notion of a multiplier ideal need not be defined. However, we overcome this difficulty by using an alternate construction of adjoint module; this adjoint module is a submodule of $\omega_{S}$ rather than an ideal of $S$, agrees with the adjoint ideal for local Gorenstein rings after fixing an isomorphism $\omega_{S} \cong S$, but seems better suited for working on singular varieties. ${ }^{2}$ In this more general setting, we show that $D$ has a section if

$$
\text { gradedcore }\left(I \omega_{S}\right)=\operatorname{adj}\left(I^{d} \omega_{S}\right), \quad \text { where } d=\operatorname{dim} S,
$$

holds for the ideal $I=S_{\geq n}, n \gg 0$, where gradedcore $\left(I \omega_{S}\right)$ is the intersection of the submodules $J \omega_{S}$ in $\omega_{S}$, as $J$ ranges over all homogeneous ideals $J$ whose integral closure is $I$, and adj $\left(I^{d} \omega_{S}\right)$ is the adjoint module, as defined in Remark 6.0 .7

Thus it may be possible to prove Kawamata's Conjecture by proving formulas (11) or (2) for section rings of divisors $D$ satisfying the conditions of Kawamata's hypothesis. Such section rings are very special: for example, in the Fano case above, the Rees ring $S[I t]$ turns out to be Cohen-Macaulay and even to have rational singularities; even for more general pairs, the Rees ring is $S[I t]$ still has the very strong property that its irrelevant ideal is a Cohen-Macaulay module. Under the strong conditions imposed by the geometric hypothesis, we hope to eventually prove formula (2) and hence Kawamata's Conjecture. What we do in the current paper is a local version of just that.

\footnotetext{
${ }^{2}$ The point is that its definition does not require the existence of a relative canonical divisor.
} 
1.3. The Main Algebraic Results. Guided by the conjectural formulas (11) and (2), we prove formulas for the core of an ideal $I$ in a local ring $A$ satisfying hypotheses satisfied by the ideal $I=S_{\geq n}$ in the special section rings $S$ arising from a pair $(X, D)$ satisfying the assumptions of Conjecture1.1.1 For example, a special case of our main theorem produces the following higher dimensional (and singular) version of the Huneke-Swanson formula for core mentioned in the opening paragraph:

Theorem 1.3.1. Let $(A, \mathfrak{m})$ be a Gorenstein local ring of dimension d essentially of finite type over a field of characteristic zero, and let I be an $\mathfrak{m}$-primary ideal of $A$. If the Rees ring $A[I t]$ of I has rational singularities, then

$$
\operatorname{core}(I)=\operatorname{adj}\left(I^{d}\right),
$$

where $\operatorname{adj}\left(I^{d}\right)$ denotes the adjoint (or multiplier) ideal of the ideal $I^{d}$.

Although the assumption above that the Rees ring has rational singularities is quite strong, it is quite natural for two reasons. First, it is satisfied when $S$ is the section ring of the anti-canonical divisor on a Fano variety and $I=S_{\geq n}$, $n \gg 0$. Thus Theorem 1.3.1 can be viewed as a "local version" of (11) and hence a local version of (a special case of) Kawamata's Conjecture. Second, it always holds whenever $I$ is an integrally closed ideal in a two-dimensional regular local ring, the setting of the Huneke-Swanson formula. In fact, we also show that under some further restrictions, rational singularities of $A[I t]$ is necessary and sufficient for formula (3) to hold (see Theorem 5.3.1).

Passing away from Gorenstein (and even Cohen-Macaulay) case, as we must in order to prove Kawamata's Conjecture in full generality, we arrive at a similar result inside the canonical module:

Theorem 1.3.2. Let $(A, \mathfrak{m})$ be a local ring essentially of finite type over a field of characteristic zero, and let $I$ be an $\mathfrak{m}$-primary ideal such that the irrelevant ideal of the Rees ring $A[I t]$ is a Cohen-Macaulay $A[I t]-$ module. Then if $Y=\operatorname{Proj} A[I t]$ has rational singularities,

$$
\operatorname{core}\left(I \omega_{A}\right)=\operatorname{adj}\left(I^{d} \omega_{A}\right):=\Gamma\left(Y, I^{d} \omega_{Y}\right)
$$

as submodules of $\omega_{A}$ where $d$ is the dimension of $A$.

Theorem 1.3.2 then, can be interpreted as a local version of (2), and so a local analog Kawamata's Conjecture. Indeed, its hypothesis is precisely satisfied by a section ring $S$ of a pair $(X, D)$ satisfying the hypothesis of Conjecture 1.1.1 Therefore, for such a section ring, Theorem 1.3 .2 implies that

$$
\operatorname{core}\left(I \omega_{S}\right)=\operatorname{adj}\left(I^{d} \omega_{S}\right)
$$

where $I=S_{\geq n}$ is the ideal generated by the elements of degrees at least $n \gg 0$ and $d$ is the dimension of $S$. We conjecture that furthermore

$$
\operatorname{gradedcore}\left(I \omega_{S}\right)=\operatorname{adj}\left(I^{d} \omega_{S}\right)
$$

holds, which, as we show in Section 6, implies Conjecture 1.1.1 The difference between (4), which we prove to be true, and (5), which implies Kawamata's Conjecture, is that in the latter we are intersecting over only homogeneous ideals $J$. On the other hand, since both intersections are actually finite and there are plenty of homogeneous reductions, it is reasonable to expect that perhaps equality holds. This, however, seems to be a very subtle question. 
Our formulas for core also elucidate the relationship between the core and the coefficient ideals of Aberbach and Huneke (see Corollary 5.4.1), and support some very general conjectures of Corso, Polini and Ulrich, which built on the HunekeSwanson work; see [7. For example, we prove:

Theorem 1.3.3. Let $(A, \mathfrak{m})$ be a Cohen-Macaulay local ring containing the rational numbers and let $I$ be any equimultiple ideal of positive height. If the Rees algebra $A[I t]$ is Cohen-Macaulay, then

$$
\text { core } I=J^{r+1}: I^{r} \quad \text { for } r \gg 0,
$$

where $J$ is any minimal reduction of $I$.

This formula for core is conjectured in more general settings in [7].

The format of the paper is as follows. Section 2 summarizes some background material and conventions, while recording various technical lemmas for later use. Section 3 forms the technical heart of this paper. Here we prove our main technical theorem, Theorem 3.0 .2 describing the core module core $\left(I \omega_{A}\right)$ of an ideal in the canonical module as an "adjoint-type" module. Theorem 3.0 .2 requires a certain Briançon-Skoda-type hypothesis, and in Section 4, we identify some natural classes of rings and ideals for which these hypotheses are satisfied. In Section 5, we pull together these results to deduce our main local results, including Theorems 1.3.1 and 1.3.3. Finally, in Section 6, we show how Kawamata's Conjecture reduces to a purely algebraic statement about the graded core analogous to our main results in the local case.

Throughout this paper, all rings and schemes are assumed Noetherian, and are assumed to possess a dualizing complex. The notation $(A, \mathfrak{m})$ denotes a local ring whose unique maximal ideal is named $\mathfrak{m}$. For an affine scheme $\operatorname{Spec} A$, we frequently abuse terminology by deliberating blurring the difference between a quasi-coherent sheaf of modules on $\operatorname{Spec} A$ and the corresponding $A$-module of its global sections.

\section{Algebraic Preliminaries.}

This section summarizes some definitions, tools and conventions we will use. With the exception of the definition of core and graded core in Subsection 1 and a few technical results (likely to be well-known to experts), nearly all of this material can be unearthed from the sources [19], 17], and [34]. Readers may prefer to skip this section and refer back only as necessary.

2.1. Integral Closure, Reductions, and the Core. Let $I$ be an ideal in a Noetherian ring $A$. The integral closure of $I$ is defined as the set of all elements $z$ in $A$ satisfying a polynomial equation

$$
z^{n}+a_{1} z^{n-1}+\cdots+a_{n}=0
$$

where $a_{i} \in I^{i}$. The integral closure $\bar{I}$ of $I$ is an ideal of $A$ containing $I$ and contained in the radical of $I$. In the case where $I$ is a homogeneous ideal of a graded ring, $\bar{I}$ is also homogeneous.

A reduction of $I$ is any sub-ideal of $I$ having the same integral closure. Equivalently, $J$ is a reduction of $I$ if there is a positive integer $r$ such that

$$
I^{r+1}=J I^{r} .
$$


The smallest such $r$ is called the reduction number of the pair $(I, J)$. Equivalently, a sub-ideal $J$ is a reduction if and only if the corresponding inclusion of Rees Rings

$$
A \oplus J \oplus J^{2} \cdots \subset A \oplus I \oplus I^{2} \ldots
$$

is integral.

A minimal reduction of an ideal $I$ is a reduction that does not properly contain any smaller reduction of $I$. Every ideal admits minimal reductions. If $I$ is an $\mathfrak{m}-$ primary ideal in a local ring $(A, \mathfrak{m})$ of dimension $d$, then any reduction generated by $d$ elements is a minimal reduction of $I$. Conversely, if the residue field $A / \mathfrak{m}$ is infinite, then every minimal reduction is generated by $d$ elements.

See [19] $\S 4, \S 10]$ for more information on integral closures and reductions.

Definition 2.1.1. The core of an ideal is the intersection of all its reductions. If the ideal is a homogeneous ideal in a graded ring, then its graded core is the intersection of all its homogeneous reductions.

Remark 2.1.2. Obviously, core $I \subset$ gradedcore $(I)$ for any homogeneous ideal in a graded ring, but the inclusion can be strict in general, even when $I$ has many homogeneous reductions; see Remark 6.4.2

Our main interest from the point of view of Kawamata's conjecture is the ideal of elements of degrees at least $n$ in a graded ring. In this case we have the following simple descriptions of integral closure, reductions, and the graded core.

Proposition 2.1.3. Let $S$ be an $\mathbb{N}$-graded reduced ring finitely generated over an infinite field $S_{0}=k$. Suppose that the set of elements of $S$ of degree $n$ generate a cofinite ideal I (that is, that $S / I$ is finite dimensional over $k$ ). Then

(1) $\bar{I}=S_{\geq n}$, the ideal generated by all elements of degrees at least $n$.

(2) Every minimal homogeneous reduction of $I$ is generated by a system of parameters for $S$ consisting of elements of degree $n$.

(3) The graded core of $\bar{I}$ is the intersection of all homogeneous systems of parameters consisting of elements of degree $n$.

Proof. Suppose that $I$ is generated by elements of degree $n$ and let $w$ be a homogeneous element of $\bar{I}$. Then $w$ satisfies a homogeneous equation of integral dependence

$$
w^{t}+a_{1} w^{t-1}+\cdots+a_{t-1} w+a_{t}=0
$$

where $a_{i} \in I^{i}$. Since $I^{i}$ is generated by elements of degree $i n$, we see that the degree of $w$ must be at least $n$ : otherwise the homogeneity forces all $a_{i}=0$ and so $w$ would be nilpotent. Thus no element of degree less than $n$ can be in $\bar{I}$.

On the other hand, fix any system of parameters $\left\{x_{1}, \ldots, x_{d}\right\}$ consisting of elements of degree $n$. Then we have a finite integral extension of graded rings

$$
A=k\left[x_{1}, \ldots, x_{d}\right] \hookrightarrow S,
$$

and so every homogeneous element $w$ of $S$ satisfies a homogeneous equation of integral dependence

$$
w^{t}+a_{1} w^{t-1}+\cdots+a_{t-1} w+a_{t}=0,
$$

where $a_{i} \in A$. Now if $w$ has degree at least $n$, the elements $a_{i}$ have degree at least in. Thus $a_{i} \in\left(x_{1}, \ldots, x_{d}\right)^{i}$, and $w$ is integral over $\left(x_{1}, \ldots, x_{d}\right)$. 
Let $J$ be any minimal homogeneous reduction of $I$ (or of $\bar{I}$ ). From the above arguments, $J$ is necessarily generated by degree $n$ elements, and since the ground field is infinite, these generators form a system of parameters. This completes the proof.

2.2. Canonical sheaves and trace. A general reference for the material in this section is [17. See also [5].

A canonical sheaf for a Noetherian scheme of dimension $d$ is defined to be the coherent sheaf given by the $-d$-th cohomology of a normalized dualizing complex for the scheme, when such a dualizing complex exists. When the scheme is CohenMacaulay, the canonical sheaf is a dualizing sheaf in the sense of Grothendieck. Dualizing complexes exist for any equidimensional scheme essentially of finite type over an affine Gorenstein scheme; see [17. p299, p306].

The canonical sheaf of a scheme $Y$ is not uniquely determined up to isomorphism in general. However, in many situations, there is a canonical choice for the canonical sheaf. For example, if $Y$ is a normal algebraic variety, then the usual notion of the canonical sheaf (namely, the unique reflexive sheaf that agrees with the sheaf of top differential Kähler forms on the smooth locus; see (6.1) provides a natural, "truly canonical" choice for $\omega_{Y}$. More generally, when all schemes are equidimensional and essentially of finite type over a fixed ground $\operatorname{scheme} \operatorname{Spec} A$ that admits a residual complex, there is a functorial procedure ("upper shriek," denoted "!") for constructing natural dualizing complexes on them from the given one on $A$. In this paper, all schemes will be of essentially finite type over a fixed local ground scheme possessing a dualizing complex (a field in the geometric settings), with respect to which all canonical sheaves will be constructed.

For affine schemes, we also use the terminology canonical module. The canonical module of a local ring $(A, \mathfrak{m})$ can be described as a finitely generated module whose dual is the top local cohomology module $H_{\mathfrak{m}}^{d}(A)$, where $d=\operatorname{dim} A$ (with the duality as described in Subsection 2.3).

2.2.1. The Canonical sheaf and Cohen-Macaulayness. The canonical sheaf of a Cohen-Macaulay scheme is itself a Cohen-Macaulay sheaf of the same dimension. Even for non-Cohen-Macaulay schemes, the canonical sheaf retains a bit of its Cohen-Macaulayness: the canonical sheaf satisfies Serre's $S_{2}$ condition. In particular, the canonical sheaf of a scheme $Y$ of dimension at most two is a CohenMacaulay sheaf, even if $Y$ itself is not Cohen-Macaulay. The basic properties of canonical modules over (non-Cohen-Macaulay) local rings are summarized, with references, in [20, Section 2].

2.2.2. Trace. Fix a proper map

$$
Y \stackrel{f}{\longrightarrow} \operatorname{Spec} A,
$$

where Spec $A$ is an equidimensional local scheme of dimension $d$ with fixed normalized residual complex $\mathcal{R}^{\bullet}$. Letting $\mathcal{R}_{Y}^{\bullet}:=f^{!} \mathcal{R}$. be the corresponding normalized residual complex for $Y$, Grothendieck's general theory of trace provides a map

$$
\mathbf{R} \Gamma\left(Y, \mathcal{R}_{Y}^{\bullet}\right) \longrightarrow \mathcal{R}^{\bullet}
$$

see [17, p318] for details on $f^{!}$and [17, p383] for more on trace. In particular, when $A$ and $Y$ have the same dimension $d$, we can take the $-d$-th cohomology, and we 
get a natural map of $A$-modules

$$
\Gamma\left(Y, \omega_{Y}\right) \longrightarrow \omega_{A},
$$

which we call the trace map. The dual of the trace map is the natural map of local cohomology modules

$$
H_{\mathfrak{m}}^{d}(A) \longrightarrow H_{Z}^{d}\left(\mathcal{O}_{Y}\right)
$$

where $\mathfrak{m}$ is the unique maximal ideal of $A$ and $Z=f^{-1}(\{\mathfrak{m}\})$ (with the duality as discussed below in Subsection 2.3.

The most important case for us is when $Y \longrightarrow \operatorname{Spec} A$ is proper and birational, specifically, a blowup along an ideal in Spec $A$. In this case, the trace map turns out to be injective; dually, the natural map of local cohomology modules $H_{\mathfrak{m}}^{d}(A) \longrightarrow$ $H_{Z}^{d}\left(\mathcal{O}_{Y}\right)$ is surjective. See, for example, [36] p103]. Throughout this paper, we will always identify $\Gamma\left(Y, \omega_{Y}\right)$ with a submodule of $\omega_{A}$ using this trace morphism when $Y \longrightarrow \operatorname{Spec} A$ is projective and birational.

In geometric situations, the meaning of the trace map is often clear. For example, if both $Y$ and Spec $A$ are normal and $Y \stackrel{f}{\longrightarrow}$ Spec $A$ is the blowing up of some closed subscheme $W \subset$ Spec $A$ of codimension at least 2, then there is a natural inclusion

$$
\Gamma\left(Y, \omega_{Y}\right) \hookrightarrow \omega_{A}
$$

obtained by restricting a global section of $\omega_{Y}$ to $Y \backslash f^{-1}(W)=\operatorname{Spec} A \backslash W$ and then extending uniquely to a section of $\omega_{A}$.

2.2.3. Adjunction. Let $E$ be a subscheme of a Cohen-Macaulay scheme $Y$ defined locally by a nonzerodivisor. Let $\mathcal{O}_{Y}(-E)$ denote the ideal sheaf of $E$, so that $\mathcal{O}_{Y}(E)$ denote its dual. Then the "upper shriek" construction in which a canonical module on $Y$ determines one on $E$ is easy to describe. Explicitly,

$$
\omega_{E}:=\mathcal{E} x t_{\mathcal{O}_{Y}}^{1}\left(\mathcal{O}_{E}, \omega_{Y}\right) \text {. }
$$

In particular, $\omega_{Y}$ and $\omega_{E}$ are related by the following exact sequence

$$
0 \longrightarrow \omega_{Y} \longrightarrow \omega_{Y}(E) \longrightarrow \omega_{E} \longrightarrow 0,
$$

obtained from the long exact sequence that arises by applying the functor $\mathcal{H}_{o m_{\mathcal{O}_{Y}}}\left(-, \omega_{Y}\right)$ to the exact sequence $0 \longrightarrow \mathcal{O}_{Y}(-E) \longrightarrow \mathcal{O}_{Y} \longrightarrow \mathcal{O}_{E} \longrightarrow 0$.

If $Y$ fails to be Cohen-Macaulay but is proper over a local scheme, and $E$ is locally defined by a nonzerodivisor, then we still have an exact sequence

$$
0 \longrightarrow \omega_{Y} \longrightarrow \omega_{Y}(E) \longrightarrow \omega_{E},
$$

but exactness on the right can fail in general.

2.3. Duality. Throughout this paper, the term "duality" always refers to the following version of Grothendieck duality combining global and local duality as developed in [17. For a careful proof of this form of duality, see [33, p188].

Let $(A, \mathfrak{m})$ be a local ring that is a homomorphic image of a Gorenstein ring (for example, essentially of finite type over a field). Let $Y \stackrel{f}{\longrightarrow}$ Spec $A$ be a proper morphism and let $Z=f^{-1}(\{\mathfrak{m}\})$ denote its closed fiber. If $Y$ is Cohen-Macaulay of equidimension $d$, then for any coherent $\mathcal{O}_{Y}$-module $\mathcal{F}$, there exist $A$-module isomorphisms for all $i$

$$
H_{Z}^{i}(Y, \mathcal{F}) \stackrel{\cong}{\longrightarrow} \operatorname{Hom}_{A}\left(\operatorname{Ext}_{Y}^{d-i}\left(\mathcal{F}, \omega_{Y}\right), E_{A}(A / \mathfrak{m})\right),
$$


where $E_{A}(A / \mathfrak{m})$ is an injective hull of the residue field of $A$, and $H_{Z}^{i}(Y, \mathcal{F})$ denotes the local cohomology module of $\mathcal{F}$ with supports in $Z$. In particular, if $\mathcal{F}$ is invertible, then

$$
H_{Z}^{i}(Y, \mathcal{F}) \quad \text { is dual to } \quad H^{d-i}\left(Y, \mathcal{F}^{-1} \otimes \omega_{Y}\right) .
$$

Note that this duality includes Serre duality as a special case. Indeed, if $Y$ is a projective variety over a field $k$, then applied to the proper map $Y \longrightarrow \operatorname{Spec} k$ (so that $Y=Z$ and $E_{A}(A / \mathfrak{m})=k$ ), we recover the standard statement of Serre duality (as in [18, p243]). At the other extreme, taking $f$ to be the identity map $\operatorname{Spec} A \longrightarrow \operatorname{Spec} A$, we recover the standard local duality familiar to commutative algebraists (as in [4 p133]).

When $Y$ is not Cohen-Macaulay, the isomorphism (6) holds as stated only for $i=d$. To get duality for all $i$, one must replace $\omega_{Y}$ in the statement of (6) by the normalized dualizing complex for $Y$. We will not need such general formulations of duality in this paper. See [33, p188].

2.4. Rees Rings and Associated Graded Rings. Let $I$ be an ideal in a Noetherian ring $A$. The Rees ring of $A$ with respect to $A$ is the $\mathbb{N}$-graded ring

$$
A[I t]:=A \oplus I \oplus I^{2} \oplus I^{3} \oplus \ldots,
$$

and the associated graded ring or form ring is the $\mathbb{N}$-graded ring

$$
\operatorname{gr}_{I} A:=A / I \oplus I / I^{2} \oplus I^{2} / I^{3} \oplus I^{3} / I^{4} \oplus \ldots
$$

In both cases, the "multiplication" is the one naturally induced by multiplication in $A$. If the ideal $I$ has positive height, then the Rees ring has dimension $d+1$, where $d=\operatorname{dim} A$, and the associated graded ring has dimension $d$. (See e.g. [19, Theorem 9.7]).

Now let $R$ and $G$ be the Rees and associated graded rings, respectively, for $A$ with respect to some ideal $I$ of positive height. Set $Y=\operatorname{Proj} R$. By definition, the natural projection $Y \stackrel{\pi}{\longrightarrow} \operatorname{Spec} A$ (induced by the inclusion of $A$ in $R$ ) is the blowing up morphism of the ideal $I$. The ideal sheaf $I \mathcal{O}_{Z}$ is invertible, and defines the scheme theoretic pre-image of the subscheme of $\operatorname{Spec} A$ defined by $I$. Thus this pre-image is a divisor, $E$, called the exceptional divisor ${ }^{3}$ of $\pi$. The natural isomorphism $R \otimes_{A} A / I \longrightarrow G$ identifies $E$ with $\operatorname{Proj} G$, so there is a fiber diagram

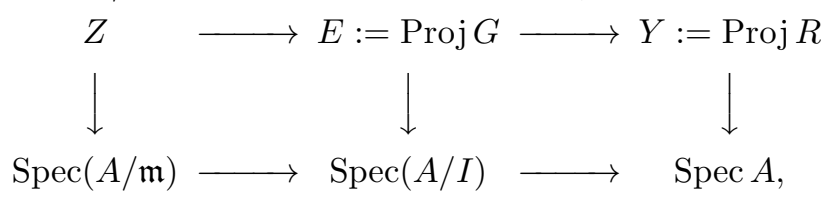

where $Z$ is the scheme-theoretic fiber over the closed point $\mathfrak{m}$ of $\operatorname{Spec} A$. In this diagram, the horizontal maps are all closed embeddings whereas the vertical maps are all proper. If $I$ is an $\mathfrak{m}$-primary ideal, the schemes $Z$ and $E$ share the same reduced subscheme. The invertible sheaves $I^{n} \mathcal{O}_{Y}$ can be identified with the coherent sheaves $\mathcal{O}_{Y}(n)$ arising from the graded $R$-modules $R(n)$ (where $R(n)_{m}=R_{n+m}$ ). This justifies our use of the notations

$$
I^{n} \mathcal{O}_{Y}, \quad \mathcal{O}_{Y}(n), \text { and } \mathcal{O}_{Y}(-n E)
$$

interchangeably, even when $n$ is negative.

\footnotetext{
${ }^{3}$ Caution: If $I$ has height one, the actual exceptional set for the map $\pi$ may not be a divisor at all, but a proper subset of $E_{r e d}$.
} 
2.4.1. Arbitrary Filtrations. More generally, Rees rings and associated graded rings can be defined with respect to an arbitrary filtration of a Noetherian ring $A$. Let $\left\{I_{n}\right\}_{n \in \mathbb{N}}$ be a filtration of $A$, that is, a descending sequence of ideals satisfying $I_{n} I_{m} \subset I_{n+m}$ for all $n, m \in \mathbb{N}$ and $I_{0}=A$. Then the Rees ring and associated graded rings are defined by

$$
A \oplus I_{1} \oplus I_{2} \oplus \ldots \quad \text { and } \quad A / I_{1} \oplus I_{1} / I_{2} \oplus I_{2} / I_{3} \oplus \ldots,
$$

respectively. The standard Rees and associated graded rings of the previous paragraph correspond to the filtration $\left\{I_{n}\right\}=\left\{I^{n}\right\}$. In general, the Rees ring and associated graded ring of an arbitrary filtration need not be Noetherian. However, if they are Noetherian and $I_{1}$ has positive height, then the dimensions are $d+1$ and $d$, respectively, where $d$ is the dimension of $A$, just as for filtrations by powers of ideals.

Given any filtration $\left\{I_{n}\right\}$ and a fixed natural number $k$, there is a Veronese subfiltration whose $n$-th member is $I_{k n}$. In this case, the corresponding Rees ring is the $k$-th Veronese subring of the Rees ring for $\left\{I_{n}\right\}$. (The affect on the associated graded ring is more subtle.) Because every finitely generated graded algebra has a Veronese sub-ring generated in degree one, any filtration giving rise to a finitely generated Rees ring admits a Veronese sub-filtration consisting of powers of an ideal $I_{k}$.

The only type of filtration we use in this paper (other than powers of $I$ ) is the "natural" filtration in a graded ring: If $S$ is a Noetherian $\mathbb{N}$-graded ring, then set $I_{n}=S_{\geq n}$ to be the ideal generated by elements of degrees at least $n$. In this case, the Rees ring, denoted $S^{\natural}$, is Noetherian and the associated graded ring is naturally isomorphic to $S$.

2.4.2. The a-invariant. Let $\mathcal{R}$ be an arbitrary Noetherian graded ring over a local ring, and let $\mathfrak{M}$ denote its unique homogeneous maximal ideal. The a-invariant of $\mathcal{R}$ is defined as

$$
a(\mathcal{R})=\max _{n}\left\{\left[H_{\mathfrak{M}}^{d}(\mathcal{R})\right]_{n} \neq 0\right\}
$$

The a-invariant of a Rees ring is always -1 while the $a$-invariant of the associated graded ring carries subtle information about the singularities of $A$ and $R$ (see 2.5 .2 below). The term "a-invariant" is due to Goto and Watanabe; see [14].

\subsection{The Sancho de Salas Sequence. Let}

$$
R=R_{0} \oplus R_{1} \oplus R_{2} \oplus \ldots
$$

be an arbitrary graded ring over a ring $R_{0}=A$, and let $\mathfrak{m}$ be an arbitrary ideal of $A$. Set $Y=\operatorname{Proj} R$ and $Z=Y \times_{\operatorname{Spec} A} \operatorname{Spec} A / \mathfrak{m}$. Then for any graded $R$-module $N=\bigoplus_{n \in \mathbb{Z}} N_{n}$, there is a degree-preserving long exact sequence:

$$
\ldots \longrightarrow H_{\mathfrak{m}_{R}}^{i}(N) \longrightarrow \bigoplus_{n \in \mathbb{Z}} H_{\mathfrak{m}}^{i}\left(N_{n}\right) \longrightarrow \bigoplus_{n \in \mathbb{Z}} H_{Z}^{i}\left(Y, \mathcal{N}_{n}\right) \longrightarrow H_{\mathfrak{m}_{R}}^{i+1}(N) \longrightarrow \ldots
$$

where $\mathfrak{m}_{R}=\mathfrak{m} \oplus R_{1} \oplus R_{2} \oplus \ldots$ and $\mathcal{N}_{n}$ denotes the quasi-coherent $\mathcal{O}_{Y}$-module corresponding to the graded $R$-module $N(n)$. This very useful sequence was introduced in [38] in a special case, and later developed by Lipman in [34. 
2.5.1. Local vs Global Cohomology. For example, consider the extreme case where $\mathfrak{m}=0$. Then $Y=Z$ and because $H_{0}^{i}(N)=0$ for $i>0$, the Sancho de Salas sequence degenerates to the long exact sequence

$$
0 \longrightarrow H_{R_{>0}}^{0}(N) \longrightarrow N \longrightarrow \bigoplus_{n \in \mathbb{Z}} H^{0}\left(Y, \mathcal{N}_{n}\right) \longrightarrow H_{R_{>0}}^{1}(N) \longrightarrow 0,
$$

where $R_{>0}=R_{1} \oplus R_{2} \oplus \ldots$ is the "irrelevant ideal" of the graded ring $R$, and the graded isomorphisms

$$
\bigoplus_{n \in \mathbb{Z}} H^{i}\left(Y, \mathcal{N}_{n}\right) \cong H_{R>0}^{i+1}(N) \quad \text { for } i \geq 1 .
$$

This is the familiar identification between sheaf cohomology on a projective scheme and the corresponding local cohomology with supports in the irrelevant ideal.

2.5.2. The case of Rees rings. Let $R$ be the Rees ring of a local ring $(A, \mathfrak{m})$ of dimension $d$ with respect to a Noetherian filtration of ideals $I_{n}$ of positive height. In this case, $\mathfrak{m}_{R}=\mathfrak{m} \oplus R_{1} \oplus R_{2} \oplus \ldots$ is the unique homogeneous maximal ideal $\mathfrak{M}_{R}$ of $R$. For the case $N=R$, the Sancho de Salas sequence is

$$
\ldots \longrightarrow H_{\mathfrak{M}_{R}}^{i}(R) \longrightarrow \bigoplus_{n \in \mathbb{N}} H_{\mathfrak{m}}^{i}\left(I_{n}\right) \longrightarrow \bigoplus_{n \in \mathbb{Z}} H_{Z}^{i}\left(Y, \mathcal{O}_{Z}(n)\right) \longrightarrow H_{\mathfrak{M}_{R}}^{i+1}(R) \longrightarrow \ldots
$$

This exact sequence can be used to quickly deduce many useful well-known facts, including:

(1) As graded $A$-modules, $H_{\mathfrak{m}_{R}}^{d+1}(R) \cong \oplus_{n<0} H_{Z}^{d}\left(Y, \mathcal{O}_{Y}(n)\right)$. This is because the maps $H_{m}^{d}\left(I_{n}\right) \longrightarrow H_{Z}^{d}\left(Y, \mathcal{O}_{Y}(n)\right)$ are surjective for all $n \geq 0$ (see, e.g. 36. p. 103].)

(2) If $R$ is Cohen-Macaulay, then $H_{Z}^{i}\left(Y, \mathcal{O}_{Y}(n)\right)=0$ for all $n<0$ and all $i<d$. By duality, this is the same as $H^{i}\left(Y, \omega_{Y}(n)\right)=0$ for all $n>0$ and all $i>0$.

(3) If $I$ is m-primary and both $A$ and $R$ are Cohen-Macaulay, then also $H_{Z}^{i}\left(Y, \mathcal{O}_{Y}(n)\right)=$ 0 for all $n \geq 0$ and all $1<i<d$. The dual statement is $H^{i}\left(Y, \omega_{Y}(n)\right)=0$ for all $n \leq 0$ and all $0<i<d-1$.

(4) If $A$ is Cohen-Macaulay, then $R$ is Cohen-Macaulay if and only if $G$ is Cohen-Macaulay with negative $a$-invariant. 13, 34

(5) If $G$ is Cohen-Macaulay, then $H_{Z}^{i}\left(Y, \mathcal{O}_{Y}\right)=0$ for all $i<d$. Dually, $H^{i}\left(Y, \omega_{Y}\right)=0$ for all $i>0$. [38, 34]

To deduce the above statements involving Cohen-Macaulayness, use the fact that a module $M$ over a local (or graded) $\operatorname{ring}(\mathcal{R}, \mathcal{M})$ is Cohen-Macaulay if and only if the local cohomology modules $H_{\mathcal{M}}^{i}(M)$ vanish for all $i<\operatorname{dim} M$.

2.6. The Graded Canonical Module. Let $R=\bigoplus_{n \in \mathbb{N}} R_{n}$ be an $\mathbb{N}$-graded ring finitely generated over a local ring $R_{0}=A$, where $(A, \mathfrak{m})$ is a homomorphic image of a Gorenstein local ring. Let $\mathfrak{M}_{R}=\mathfrak{m} \oplus R_{1} \oplus R_{2} \oplus \ldots$ denote the unique homogeneous maximal ideal of $R$. Then $R$ admits a graded canonical module, which by definition, is a finitely generated graded $R$-module such that

$$
\underline{\operatorname{Hom}}_{A}\left(\omega_{R}, E_{A}(A / \mathfrak{m})\right) \cong H_{\mathfrak{M}_{R}}^{\operatorname{dim} R}(R)
$$


where $E_{A}(A / \mathfrak{m})$ is an injective hull of the $A$-module $A / \mathfrak{m}$ and the notation $\underline{\text { Hom }}$ denotes "graded homomorphisms", namely

$$
\underline{\operatorname{Hom}}_{A}\left(\omega_{R}, E_{A}(A / \mathfrak{m})\right)=\bigoplus_{n \in \mathbb{Z}} \operatorname{Hom}_{A}\left(\left[\omega_{R}\right]_{-n}, E_{A}(A / \mathfrak{m})\right) .
$$

In other words, a graded canonical module is a finitely generated graded $R$-module whose "graded Matlis dual" is isomorphic, as a graded $R$-module, to the top local cohomology module with supports in the unique homogeneous maximal ideal of $R$. The graded canonical module is uniquely determined up to degree preserving homomorphism. Furthermore, it is a canonical module for $R$ in the non-graded sense as well. For details and generalities on graded canonical modules and related material, see 19, Chapter VII, especially Section 36, or [4, Section 3.6, or the original paper of Goto and Watanabe [14].

2.6.1. The canonical module for rings graded over a field. Let $S$ be an $\mathbb{N}$-graded ring over a field $k=A$, and let $X=\operatorname{Proj} S$ be the corresponding projective scheme. By definition, the graded pieces of the graded canonical module $\omega_{S}$ are dual to the graded pieces of $H_{\mathfrak{M}_{S}}^{\operatorname{dim}} S(S)$. In the case where $X$ has dimension at least one (so $S$ has dimension at least two), this latter module can be identified with (see 2.5.1)

$$
\bigoplus_{n \in \mathbb{Z}} H^{\operatorname{dim} X}\left(X, \mathcal{O}_{X}(n)\right) \text {. }
$$

So, we use duality (which holds at the top spot even when $X$ is not CohenMacaulay) to conclude that

$$
\omega_{S}=\bigoplus_{n \in \mathbb{Z}} H^{0}\left(X, \omega_{Y}(n)\right)
$$

is a graded canonical module for $S$.

2.6.2. The canonical module for $R$. In the case where $R$ is a Rees ring of an ideal of positive height in a local ring $(A, \mathfrak{m})$, we have

$$
\omega_{R}=\bigoplus_{n>0} H^{0}\left(Y, \omega_{Y}(n)\right),
$$

where $\omega_{Y}$ is the canonical module on $Y=\operatorname{Proj} R$ constructed from the fixed one on $A$. Indeed, its dual is $\bigoplus_{n<0} H_{Z}^{d}\left(Y, \mathcal{O}_{Y}(-n)\right)$, which is identified with $H_{\mathfrak{M}_{R}}^{d+1}(R)$ as a graded module, by 2.5.2 (1). More generally, this argument shows that for any graded ring $R$ over a local ring $A,\left[\omega_{R}\right]_{n}=H^{0}\left(Y, \omega_{Y}\right) n$ ) for positive $n$, where $Y=\operatorname{Proj} R$.

2.6.3. The canonical module for $G$. Likewise, there is a similar choice for the associated graded ring $G$ of an $\mathfrak{m}$-primary ideal, at least when $d \geq 2$, namely

$$
\omega_{G}=\bigoplus_{n \in \mathbb{Z}} H^{0}\left(E, \omega_{E}(n)\right)
$$

where $\omega_{E}$ is the canonical module on $E=\operatorname{Proj} G$ constructed from the fixed one on $A$. Indeed, its dual is $\bigoplus_{n \in \mathbb{Z}} H^{d-1}\left(E, \mathcal{O}_{E}(-n)\right)$, which is identified with $H_{\mathfrak{M}_{G}}^{d}(G)$ as a graded module, by the identity (2.5.1) above. In dimension one, these arguments give only that

$$
\left[\omega_{G}\right]_{n}=H^{0}\left(E, \omega_{E}(n)\right)
$$


for positive $n$. The non-positive pieces of $\omega_{G}$ are more complicated to describe (and are of crucial importance in our arguments). These will be treated later in Lemma 3.2 .1

2.6.4. The effect of killing a parameter. Let $\omega_{G}$ be the graded canonical module of a Cohen-Macaulay graded ring $G$ over a local ring $G_{0}$, and let $x$ be any homogeneous nonzerodivisor. Set $\bar{G}$ to be the graded $\operatorname{ring} G / x G$. Then

$$
\omega_{\bar{G}} \cong \frac{\omega_{G}}{x \omega_{G}}(n),
$$

as graded modules, where $n$ is the degree of $x$. Here recall that for a graded module $M$, the notation $M(n)$ denotes the same module with the degree shifted so that $[M(n)]_{t}=[M]_{n+t}$. This is well-known and easy to prove; see, for example, 4], Corollary 3.6.14].

More generally, even when $G$ is not Cohen-Macaulay, we often have useful statements along these lines. For example, we will make use of the following proposition:

Proposition 2.6.1. Let $G$ be an $\mathbb{N}$-graded ring of dimension $d>0$, finitely generated over an Artin local ring $G_{0}$, and let $x$ be any homogeneous element of degree $n$. Then there is a natural degree-preserving injection

$$
\frac{\omega_{G}}{x \omega_{G}}(n) \hookrightarrow \omega_{\bar{G}},
$$

where $\bar{G}$ denotes the ring $G / x G$, whenever the dimension of $\operatorname{Ann}_{G}(x)$ (as a $G$ module) is strictly less $d$.

Proof. Consider the four term exact sequence of degree preserving maps

$$
0 \longrightarrow \operatorname{Ann}_{G}(x) \longrightarrow G(-n) \stackrel{x}{\rightarrow} G \longrightarrow G / x G \longrightarrow 0 .
$$

Breaking this up, the short exact sequence

$$
0 \longrightarrow \operatorname{Ann}_{G}(x) \longrightarrow G(-n) \stackrel{x}{\rightarrow} x G \longrightarrow 0
$$

induces an isomorphism

$$
H_{\mathfrak{M}_{G}}^{d}(G)(-n) \stackrel{x}{\longrightarrow} H_{\mathfrak{M}_{G}}^{d}(x G),
$$

since $H_{\mathfrak{M}_{G}}^{d}\left(\operatorname{Ann}_{G}(x)\right)=0$. So the sequence

$$
0 \longrightarrow x G \longrightarrow G \longrightarrow G / x G \longrightarrow 0
$$

gives rise to the exact sequence

$$
H_{\mathfrak{M}_{G}}^{d-1}(G / x G) \longrightarrow H_{\mathfrak{M}_{G}}^{d}(G(-n)) \stackrel{x}{\longrightarrow} H_{\mathfrak{M}_{G}}^{d}(G) \longrightarrow 0 .
$$

Dualizing (that is, applying the graded Matlis dual functor; see 2.6) yields an exact sequence

$$
0 \longrightarrow \omega_{G} \stackrel{x}{\longrightarrow} \omega_{G}(n) \longrightarrow \omega_{G / x G},
$$

which provides the natural inclusion

$$
\frac{\omega_{G}}{x \omega_{G}}(n) \hookrightarrow \omega_{\bar{G}} .
$$




\section{The Main Technical Theorem}

This section forms the technical heart of this paper. Here we prove Theorem 3.0 .2 which will later be used to deduce the higher dimensional versions of the Huneke-Swanson formulas for core, our "local version" of Kawamata's conjecture, a formula for core conjectured by Corso, Polini and Ulrich, the results linking core and coefficients ideals, and other properties of core in Section 5 and 6.4.

Recall that if $Y=\operatorname{Proj} A[I t] \longrightarrow \operatorname{Spec} A$ is the blow-up of Spec $A$ along an ideal $I$, then the $A$-module $H^{0}\left(Y, \omega_{Y}\right)$ can be naturally identified with a submodule of $\omega_{A}$ (see 2.2.2). Likewise, the modules $H^{0}\left(Y, I^{n} \omega_{Y}\right)$ for $n \geq 0$ can be identified with submodules of $\omega_{A}$, denoted by $\Omega_{n}$.

Theorem 3.0.2. Let $I$ be an $\mathfrak{m}$-primary ideal in a local ring $(A, \mathfrak{m})$ of positive dimension d containing the rational numbers. Assume that for any reduction $J$ of $I$,

$$
J \omega_{A} \cap \Omega_{d-1}=J\left(\Omega_{d-2} \cap \omega_{A}\right),
$$

as submodules of $\omega_{A}$. Then

$$
\operatorname{core}\left(I \omega_{A}\right) \subset \Omega_{d}
$$

as submodules of $\omega_{A}$, where core $I \omega_{A}$ denotes the intersection in $\omega_{A}$ of the submodules $J \omega_{A}$ as $J$ ranges through all reductions of $I$.

Remark 3.0.3. Note that if $d \geq 2$, then $\Omega_{d-2}$ is contained in $\omega_{A}$ in any case; the intersection with $\omega_{A}$ is relevant only when $d=1$.

Remark 3.0.4. For ideals $I$ of reduction number at most one, the assumption that $A$ contains the rational numbers is unnecessary; see Remark 3.4.7

Remark 3.0.5. In the geometric setting, the module $H^{0}\left(Y, I^{d} \omega_{Y}\right)$ is closely related to the adjoint ideal (or multiplier ideal) of $I^{d}$, at least when $Y$ happens to be smooth (or have rational singularities). See Remark 6.0.7

As we will see in the next section, the hypothesis (7) of Theorem 3.0 .2 is a type of "Briançon-Skoda" statement, and it is satisfied in many nice situations. For example, we will show that (7) holds whenever $A$ and the Rees ring $A[I t]$ are Cohen-Macaulay, or more generally even if $A$ is not Cohen-Macaulay provided that the irrelevant ideal of the Rees ring $A[I t]$ is a Cohen-Macaulay module. This latter condition arises naturally in the geometric setting that motivates us.

The proof of Theorem 3.0 .2 will occupy this entire section. The main point is that Lemma 3.3.1 reduces us to a related statement about the intersection of the corresponding submodules of the canonical module $\omega_{G}$ of the associated graded ring $G$. This statement about $\omega_{G}$ is then proved by induction on the dimension, with the hard part being the case where $d=1$. For all these steps, we need a rather delicate understanding of the structure of the canonical module for $G$ - especially how its graded pieces are related to the adjoint-type modules $\Omega_{n}$. Thus we begin in Subsection 3.1 with a detailed study of the modules $\Omega_{n}$. 
3.1. The filtration by Adjoint-type modules. We fix some notation to be used throughout the rest of this section. We let $(A, \mathfrak{m})$ denote a local ring of dimension $d \geq 1$ which is assumed to have an infinite residue field (and as always, possesses a canonical module). Let $I$ denote a proper ideal in $A$ of positive height. Let $R$ and $G$ denote the Rees ring and the associated graded ring with respect to $I$, respectively. We set $Y=\operatorname{Proj} R$ and let $Y \stackrel{\pi}{\rightarrow}$ Spec $A$ denote the natural blowing up morphism. As always, we identify $\pi_{*} \omega_{Y}$ with a submodule of $\omega_{A}$ (see Subsection 2.2.2).

We first establish some elementary properties of the "adjoint-type" $A$-modules

$$
\Omega_{n}:=\Gamma\left(Y, I^{n} \omega_{Y}\right)
$$

where $n \in \mathbb{Z}$. First note that:

(1) Each $\Omega_{n}$ is a finitely generated $A$-module.

(2) If $n \geq m$, then $\Omega_{n} \subset \Omega_{m}$.

(3) For all $n \in \mathbb{Z}$, we have $I \Omega_{n} \subset \Omega_{n+1}$.

(4) There are natural identifications $\Omega_{n}=\operatorname{Hom}_{A}\left(I^{p}, \Omega_{n+p}\right)$ for all $n \in \mathbb{Z}$ and all $p \geq 0$.

The first property is immediate from the properness of $Y \longrightarrow$ Spec $A$, while the next two properties follow immediately from the definition. The fourth property follows from the useful but elementary general fact: Let $\mathcal{F}$ be a coherent $\mathcal{O}_{Y}$-module such that the local generator for $I \mathcal{O}_{Y}$ is a nonzerodivisor on $\mathcal{F}$ at each point of $Y$. Then $\operatorname{Hom}_{Y}\left(J \mathcal{O}_{Y}, \mathcal{F}\right)=\operatorname{Hom}_{A}(J, \Gamma(Y, \mathcal{F}))$ for any ideal $J \subset A$. (This fact is easy to prove; see, for example, Lemma 2.1 of [24].)

Our proof of Theorem 3.0 .2 will exploit the following relationship between the $\Omega_{n}$ and the graded pieces of $\omega_{G}$.

Lemma 3.1.1 (Cf. 24], Theorem 2.2e). Let I be an $\mathfrak{m}$-primary ideal in a local ring $(A, \mathfrak{m})$ of positive dimension $d$. Then there is a natural inclusion

$$
\bigoplus_{n \geq 1} \Omega_{n-1} / \Omega_{n} \hookrightarrow \omega_{G}
$$

of graded $G$-modules. This inclusion is an isomorphism if $Y$ is Cohen-Macaulay and $H^{1}\left(Y, \omega_{Y}(n)\right)=0$ for all $n$, for example, if both $R$ and $A$ are Cohen-Macaulay.

Proof. Consider the adjunction sequence

$$
0 \longrightarrow \omega_{Y} \longrightarrow \omega_{Y}(-1) \longrightarrow \omega_{E},
$$

which is exact also on the right if $Y$ is Cohen-Macaulay. Tensoring with $\mathcal{O}_{Y}(n)$ and computing cohomology we get an exact sequence of cohomology

$$
0 \longrightarrow H^{0}\left(Y, \omega_{Y}(n)\right) \longrightarrow H^{0}\left(Y, \omega_{Y}(n-1)\right) \longrightarrow H^{0}\left(E, \omega_{E}(n)\right) .
$$

Thus there is a natural injection

$$
\bigoplus_{n \in \mathbb{Z}} \Omega_{n-1} / \Omega_{n} \hookrightarrow \bigoplus_{n \in \mathbb{Z}} H^{0}\left(E, \omega_{E}(n)\right)
$$

for all $n \in \mathbb{Z}$. Since $\omega_{G}$ and $\bigoplus_{n \in \mathbb{Z}} H^{0}\left(E, \omega_{E}(n)\right)$ agree in positive degrees, (see 2.6.3), the first claim is proven.

If $Y$ is Cohen-Macaulay, then we have an exact sequence

$$
0 \longrightarrow H^{0}\left(Y, \omega_{Y}(n)\right) \longrightarrow H^{0}\left(Y, \omega_{Y}(n-1)\right) \longrightarrow H^{0}\left(E, \omega_{E}(n)\right) \longrightarrow H^{1}\left(Y, \omega_{Y}(n)\right),
$$


so the inclusion is a bijection in degree $n$ if $H^{1}\left(Y, \omega_{Y}(n)\right)=0$. When $R$ and $A$ are both Cohen-Macaulay, the scheme $Y=\operatorname{Proj} R$ is Cohen-Macaulay, and this vanishing holds (see 2.5.2). The lemma is proved.

Remark 3.1.2. The proof of Lemma 3.1.1 shows that if $d \geq 2$, the inclusion $\bigoplus_{n \in \mathbb{Z}} \Omega_{n-1} / \Omega_{n} \hookrightarrow \omega_{G}$ holds for all $n$, not just positive $n$. A nice consequence is that, for dimension $\geq 2$, the increasing chain of modules

$$
\cdots \subset \Omega_{2} \subset \Omega_{1} \subset \Omega_{0} \subset \Omega_{-1} \subset \Omega_{-2} \ldots
$$

must stabilize for $-n<-a$, where $a$ is the $a$-invariant of $G$. In fact, it stabilizes to $\omega_{A}$ :

Lemma 3.1.3. Let I be an $\mathfrak{m}$-primary ideal in a local ring $(A, \mathfrak{m})$ of dimension at least two. Then

$$
\Omega_{-n}=\omega_{A}
$$

for all $n>a(G)$.

Proof. Fix $n \gg 0$. To verify that $\omega_{A} \subset \Omega_{-n}$, recall that $\Omega_{-n}=\operatorname{Hom}_{A}\left(I^{n}, \Omega_{0}\right)$. So it will suffice to show that for $n \gg 0, I^{n} \omega_{A} \subset \Omega_{0}$ (then each $f$ in $\omega_{A}$ determines the element "multiplication by $f$ " in $\Omega_{-n}=\operatorname{Hom}_{A}\left(I^{n}, \Omega_{0}\right)$.) Because the blowup map restricts to an isomorphism away from the closed set $\operatorname{Spec}(A / I)$, the trace map $\Omega_{0} \subset \omega_{A}$ becomes an identity after localizing at any element of $I$. So some power of $I$ annihilates $\omega_{A} / \Omega_{0}$, and $I^{n} \omega_{A} \subset \Omega_{0}$ for large $n$, as needed.

To check the reverse inclusion, consider the exact sequence of $A$-modules

$$
0 \longrightarrow \omega_{A} \longrightarrow \Omega_{-n} \longrightarrow Q \longrightarrow 0
$$

where, because $I$ is $\mathfrak{m}$-primary, the module $Q$ is supported at $\mathfrak{m}$. There is an induced sequence of local cohomology

$$
H_{\mathfrak{m}}^{0}\left(\Omega_{-n}\right) \longrightarrow H_{\mathfrak{m}}^{0}(Q) \longrightarrow H_{\mathfrak{m}}^{1}\left(\omega_{A}\right) .
$$

The right term above vanishes because $\omega_{A}$ is $S_{2}$ while the left term vanishes because $\Omega_{-n}=\operatorname{Hom}_{A}\left(\Omega_{n}, \Omega_{0}\right) \subset \operatorname{Hom}_{A}\left(\Omega_{n}, \omega_{A}\right)$, so no element can be killed by any parameter in $A$. Thus $H_{\mathfrak{m}}^{0}(Q)=0$, and since $Q$ is supported only at $\mathfrak{m}, Q=0$ as well.

In dimension one, the picture is somewhat different: although it is still true that $\omega_{A} \subset \Omega_{-n}$ for large $n$, we do not get stabilization.

Lemma 3.1.4. If I is integral over a principle ideal, then $\Omega_{n}=x \Omega_{n-1}$ for all $n \in \mathbb{Z}$, where $x$ generates a minimal reduction for $I$. This holds in particular when $I$ is an $\mathfrak{m}$-primary ideal in a local ring of dimension one.

Proof. In this case, $Y=\operatorname{Proj} A[I t]$ is affine, so

$$
\Omega_{n}=I^{n} \omega_{Y}=x^{n} \omega_{Y}=x\left(I^{n-1} \omega_{Y}\right)=x \Omega_{n-1}
$$

for all $n$.

The next lemma refines our understanding of the modules $\Omega_{n}$ for the case of non-negative $n$. 
Lemma 3.1.5. Let $(A, m)$ be a local ring and let $I$ be a proper ideal of $A$ of height greater than one. Then $\Omega_{n+1}: \omega_{A} I=\Omega_{n}$ for all $n \geq 0$. In fact, $\Omega_{n+p}: \omega_{A} I^{p}=\Omega_{n}$ for all $n \geq 0$ and all $p \geq 1$. This also holds for ideals of height one that are integral over a principle ideal.

Proof. Recall that $\Omega_{n}=\operatorname{Hom}_{A}\left(I^{p}, \Omega_{n+p}\right)$. Clearly each element $w$ of $\Omega_{n+p}: \omega_{A}$ $I^{p}$ gives rise to an element of $\Omega_{n}$, namely, the "multiplication by $w$ map" in $\operatorname{Hom}_{A}\left(I^{p}, \Omega_{n+p}\right)$. We need to show that every $A$-module map from $I^{p}$ to $\Omega_{n+p}$ arises this way.

We treat the height greater than one case first. Because $I^{p}$ contains a regular sequence of length two on $\omega_{A}$, we have $\operatorname{Hom}_{A}\left(I^{p}, \omega_{A}\right)=\omega_{A}$. Since $\operatorname{Hom}_{A}\left(I^{p}, \Omega_{n+p}\right) \subset$ $\operatorname{Hom}_{A}\left(I^{p}, \omega_{A}\right)$, this implies that every element of $\operatorname{Hom}_{A}\left(I^{p}, \Omega_{n+p}\right)$ arises by multiplication by some element of $\omega_{A}$, as needed.

Now suppose $I$ has a reduction generated by one element, say $x$. By Lemma 3.1.4 we have $\Omega_{n+1}=x \Omega_{n}$ for all $n \in \mathbb{Z}$. So also $\Omega_{n+p}=x^{p} \Omega_{n}$ for all $n \geq 0$ and all $p \geq 1$. Take any $u \in \Omega_{n}=\operatorname{Hom}_{A}\left(I^{p}, \Omega_{n+p}\right)$. Then $u\left(x^{p}\right)=x^{p} \omega$ for some $\omega \in \Omega_{n} \subset \omega_{A}$. We claim now that $u$ is the map "multiplication by $\omega . "$ To check this, take any $a \in I^{p}$. We get $x^{p} u(a)=a u\left(x^{p}\right)=a x^{p} \omega$, so because $x^{p}$ is a nonzerodivisor on $\omega_{A}$, we conclude that $u(a)=a \omega$. This shows that $\Omega_{n}=$ $\operatorname{Hom}_{A}\left(I^{p}, \Omega_{n+p}\right) \subset \Omega_{n+p}: \omega_{A} I^{p}$. The proof is complete.

3.2. The canonical module $\omega_{G}$ in the dimension one case. Because our strategy for proving Theorem 3.0 .2 is to reduce to the one-dimensional case, we need to understand $\omega_{G}$ in the case $d=1$. In dimension one, if $R$ and $G$ are both Cohen-Macaulay, it is easy to see that

$$
\omega_{G} \cong \bigoplus_{n>0} \frac{\Omega_{n-1}}{\Omega_{n}}
$$

However, our reduction to the dimension one case will destroy the Cohen-Macaulayness of $R$ (even when it is assumed at the start), so the following lemma is crucial.

Lemma 3.2.1. Let $(A, \mathfrak{m})$ be a one-dimensional local ring. Let $I \subset A$ be an $\mathfrak{m}$ primary ideal. Then

$$
\left[\omega_{G}\right]_{n}= \begin{cases}\Omega_{n-1} / \Omega_{n} & \text { for } n>0 \\ \frac{\Omega_{n-1} \bigcap \operatorname{Hom}_{A}\left(I^{-n}, \omega_{A}\right)}{\Omega_{n}} & \text { for } n \leq 0 .\end{cases}
$$

Here, for $n \leq 0$, the intersection is carried out in $\operatorname{Hom}_{A}\left(I^{-n+1}, \omega_{A}\right)$ which naturally contains both $\Omega_{n-1}=\operatorname{Hom}_{A}\left(I^{-n+1}, \Omega_{0}\right)$ and $\operatorname{Hom}_{A}\left(I^{-n}, \omega_{A}\right)$.

To understand the lemma, note that for $n \leq 0$, the module $\Omega_{n}$ can be considered as a submodule of $\operatorname{Hom}_{A}\left(I^{-n}, \omega_{A}\right)$ via the injection $\Omega_{n}=\operatorname{Hom}_{A}\left(I^{-n}, \Omega_{0}\right) \longrightarrow$ $\operatorname{Hom}_{A}\left(I^{-n}, \omega_{A}\right)$ induced by the inclusion $\Omega_{0} \subset \omega_{A}$. Also, there is a natural inclusion of $\operatorname{Hom}_{A}\left(I^{-n}, \omega_{A}\right)$ in $\operatorname{Hom}_{A}\left(I^{-n+1}, \omega_{A}\right)$, induced as the dual of the inclusion $I^{-n+1} \subset I^{-n}$, since $\operatorname{Hom}_{A}\left(I^{-n} / I^{-n+1}, \omega_{A}\right)=0$.

Proof. Consider the long exact sequence (see 2.5.1) relating cohomology for $G$ and $\operatorname{Proj} G$ :

$$
0 \longrightarrow H_{\mathfrak{M}_{G}}^{0}(G) \longrightarrow G \longrightarrow \bigoplus_{n \in \mathbb{Z}} H^{0}\left(E, \mathcal{O}_{E}(n)\right) \longrightarrow H_{\mathfrak{M}_{G}}^{1}(G) \longrightarrow 0
$$


Applying duality for the proper map $E \longrightarrow \operatorname{Spec} A / I$, we get an exact sequence

$$
0 \longrightarrow \omega_{G} \longrightarrow \bigoplus_{n \in \mathbb{Z}} H^{0}\left(E, \omega_{E}(n)\right) \longrightarrow \bigoplus_{n \in \mathbb{Z}} \operatorname{Hom}_{A / I}\left(G_{n}, E_{A / I}\right)=E_{G},
$$

where $E_{A / I}$ denotes the injective hull of the residue field of the zero dimensional ring $A / I$ (and $E_{G}$ is by definition the graded injective hull of the residue field of $G$; see [19, p293]). For $n>0,\left[E_{G}\right]_{n}=0$, so there are natural isomorphisms

$$
\left[\omega_{G}\right]_{n}=H^{0}\left(E, \omega_{E}(n)\right)
$$

for all $n>0$, whereas for $-n \leq 0$, we have

$$
\left[\omega_{G}\right]_{-n}=\operatorname{ker}\left[H^{0}\left(E, \omega_{E}(-n)\right) \longrightarrow \operatorname{Hom}_{A}\left(I^{n} / I^{n+1}, E_{A / I}\right)\right] .
$$

Note that $Y$ is always Cohen-Macaulay: Away from $E, Y$ is isomorphic to the zero-dimensional scheme Spec $A \backslash \mathfrak{m}$, whereas since $E$ is defined by a nonzerodivisor locally in $Y$, the Cohen-Macaulay property of the zero-dimensional scheme $E$ lifts to $Y$. So we have a twisted adjunction sequence

$$
0 \longrightarrow \omega_{Y}(n) \longrightarrow \omega_{Y}(n-1) \longrightarrow \omega_{E}(n) \longrightarrow 0 .
$$

Because $Y$ is affine, we have a corresponding sequence of global sections

$$
0 \longrightarrow \Omega_{n} \longrightarrow \Omega_{n-1} \longrightarrow H^{0}\left(E, \omega_{E}(n)\right) \longrightarrow 0 .
$$

Hence $\Omega_{n-1} / \Omega_{n}=H^{0}\left(E, \omega_{E}(n)\right)$ for all $n \in \mathbb{Z}$. So for $n>0$, we conclude that

$$
\left[\omega_{G}\right]_{n}=\Omega_{n-1} / \Omega_{n}
$$

as needed.

It remains to treat the case $n \leq 0$. Because the case $n=0$ is the only one we actually need later, we write down the argument carefully only in this case for the sake of clarity. However, the same exact argument "twisted" by $\mathcal{O}(n)$ produces the result for any $n \leq 0$.

Consider the case $n=0$. Because $\omega_{A / I}$ is an injective hull of the residue field for the zero-dimensional local ring $A / I$, we have that

$$
\left[\omega_{G}\right]_{0}=\operatorname{ker}\left[H^{0}\left(E, \omega_{E}\right) \stackrel{\eta}{\rightarrow} \operatorname{Hom}_{A}\left(A / I, \omega_{A / I}\right)=\omega_{A / I}\right] .
$$

We need to understand the map $\eta$. Being dual to the natural map

$$
A / I=H_{\mathfrak{m}}^{0}(A / I) \longrightarrow H_{E}^{0}\left(\mathcal{O}_{E}\right)=\mathcal{O}_{E},
$$

the map $\eta$ is in fact the trace map for the proper map of zero dimensional schemes $E \longrightarrow \operatorname{Spec} A / I$. (Note that the functors $\operatorname{Hom}_{A}\left(-, \omega_{A / I}\right)$ and $\operatorname{Hom}_{E}\left(-, \omega_{E}\right)$ are identical on $\mathcal{O}_{E}$-modules by the adjointness of tensor and Hom.)

To understand $\eta$, we consider the commutative diagram of $A$-modules, whose existence we will justify momentarily:

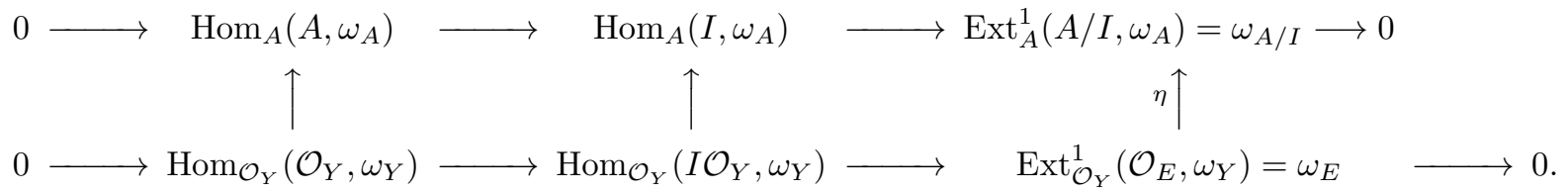

In this diagram, the first two upward arrows are injective (note the first one is the trace map for $Y \longrightarrow$ Spec $A$ ), and it is the kernel of the rightmost upward arrow 
that we want to understand. Knowing that $\left[\omega_{G}\right]_{0}=\operatorname{ker} \eta$, a look at this diagram shows that there is a natural identification

$$
\left[\omega_{G}\right]_{0}=\frac{\operatorname{Hom}_{\mathcal{O}_{Y}}\left(I \mathcal{O}_{Y}, \omega_{Y}\right) \cap \operatorname{Hom}_{A}\left(A, \omega_{A}\right)}{\operatorname{Hom}_{\mathcal{O}_{Y}}\left(\mathcal{O}_{Y}, \omega_{Y}\right)}=\frac{\Omega_{-1} \cap \omega_{A}}{\Omega_{0}},
$$

as claimed. This will complete the proof in the case where $n=0$; the proof for arbitrary negative $n$ is essentially the same, just "twisted" by $n$. It remains only to justify this commutative diagram.

Finally, to justify the diagram is easy. ${ }^{4}$ It is induced from the diagram of long exact sequences arising from the natural diagram of $A$-modules

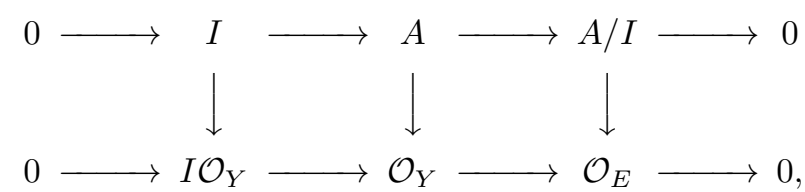

where the downward arrows are the natural inclusions. (Recall that $Y$ and $E$ are affine, so we abuse notation, identifying $\mathcal{O}_{Y}$ and $\mathcal{O}_{E}$ with their corresponding rings of global sections.) The existence of a natural induced map between the corresponding long exact Ext-sequences is a very general fact holding for any diagram of short exact sequences over any commutative rings $R \longrightarrow S$. Indeed, say $M$ and $P$ are $R$-modules, and $N$ and $Q$ are $S$-modules. Given $R$-module maps $M \longrightarrow N$ and $Q \longrightarrow P$, there are naturally induced functorial maps

$$
\operatorname{Ext}_{S}^{i}(N, Q) \longrightarrow \operatorname{Ext}_{R}^{i}(M, P)
$$

for each $i \in \mathbb{N}$. These maps can be viewed as the composition of three natural maps of $R$-modules:

$$
\operatorname{Ext}_{S}^{i}(N, Q) \longrightarrow \operatorname{Ext}_{R}^{i}(N, Q) \longrightarrow \operatorname{Ext}_{R}^{i}(N, P) \longrightarrow \operatorname{Ext}_{R}^{i}(M, P) .
$$

The first arrow above is naturally induced by the "forgetful functor." (An injective resolution $Q \longrightarrow I^{\bullet}$ of $Q$ as an $S$-module can be viewed as an exact sequence of $R$ modules; then for any injective resolution $Q \longrightarrow J^{\bullet}$ of $Q$ as an $R$-module, there will be an induced $R$-module map of complexes $I^{\bullet} \longrightarrow J^{\bullet}$. This induces an $R$-module map of complexes $\operatorname{Hom}_{R}\left(N, I^{\bullet}\right) \longrightarrow \operatorname{Hom}_{R}\left(N, J^{\bullet}\right)$, which can be pre-composed with the map that forgets the $S$-structure $\operatorname{Hom}_{S}\left(N, I^{\bullet}\right) \longrightarrow \operatorname{Hom}_{R}\left(N, I^{\bullet}\right)$. This map of complexes induces a unique map on the level of cohomology, and this is the natural map of Ext groups we have in the first arrow above.) The second arrow is the natural covariantness of the functor Ext in the second argument, and the third arrow is the natural contravariantness in the first argument.

The proof of Lemma 3.2.1 is complete, at least for $n \geq 0$. The proof for arbitrary negative $n$ is essentially the same as the proof for $n=0$, just "twisted" by $n$. (We will use this lemma only in the cases $n=0$ and $n=1$.)

3.3. Reduction to the Associated Graded Ring. We now state and prove the key lemma which provides the crucial step of reducing Theorem 3.0 .2 to a related statement about the canonical module of the associated graded ring.

\footnotetext{
${ }^{4}$ One can do this abstractly, using the point of view of [17, but we prefer a hands on verification.
} 
Lemma 3.3.1 (Key Lemma). Let I be an $\mathfrak{m}$-primary ideal in a local ring $(A, \mathfrak{m})$ of positive dimension $d$. Let $\mathcal{R}$ be any non-empty set of minimal reductions of $I$. As in Theorem 3.0.2, assume that

$$
J \omega_{A} \cap \Omega_{d-1}=J\left(\Omega_{d-2} \cap \omega_{A}\right)
$$

for all reductions $J \in \mathcal{R}$. Then

$$
\bigcap_{\left\{x_{1}, \ldots, x_{d}\right\} \in \mathcal{R}}\left(x_{1}, \ldots, x_{d}\right) \omega_{A} \subset \Omega_{d}
$$

if

$$
\bigcap_{\left\{x_{1}, \ldots, x_{d}\right\} \in \mathcal{R}}\left[\left(x_{1}^{*}, \ldots, x_{d}^{*}\right) \omega_{G}\right]_{d}=0
$$

where $x_{i}^{*}$ denotes the degree one element of $G$ given by the class of $x_{i}$ modulo $I^{2}$. The converse also holds if $Y$ is Cohen-Macaulay and both $H^{1}\left(Y, \omega_{Y}(d)\right)$ and $H^{1}\left(Y, \omega_{Y}(d-1)\right)$ vanish.

Proof. Set

$$
W=\bigcap_{\left\{x_{1}, \ldots, x_{d}\right\} \in \mathcal{R}}\left(x_{1}, \ldots, x_{d}\right) \omega_{A} .
$$

We already know that $\Omega_{d} \subset W$, because of our assumption that $\Omega_{d} \subset J \omega_{A}$ for all reductions $J$. Let $x \in W \backslash \Omega_{d}$. Since the only associated prime of $\omega_{A} / \Omega_{d}$ is $\mathfrak{m}$, we may assume that $x \in \Omega_{d}: \omega_{\omega_{A}} \mathfrak{m} \subset \Omega_{d}:_{\omega_{A}} I$. But then $x \in \Omega_{d-1}$ by Lemma 3.1.5 and its class in $\Omega_{d-1} / \Omega_{d}$ determines a non-zero element of $\left[\omega_{G}\right]_{d}$ under the natural inclusion $\Omega_{d-1} / \Omega_{d} \hookrightarrow\left[\omega_{G}\right]_{d}$ guaranteed by Lemma 3.1.1] Because $J \omega_{A} \cap \Omega_{d-1}=J\left(\Omega_{d-2} \cap \omega_{A}\right)$ for any minimal reduction $J$ of $I$ (by hypothesis), we see that

$$
x \in \bigcap_{\left\{x_{1}, \ldots, x_{d}\right\} \in \mathcal{R}}\left(x_{1}, \ldots, x_{d}\right)\left(\Omega_{d-2} \cap \omega_{A}\right) .
$$

By Remark 3.1.2 and Lemma 3.2.1 we conclude that the class of $x$ is in

$$
\bigcap_{\left\{x_{1}, \ldots, x_{d}\right\} \in \mathcal{R}}\left[\left(x_{1}^{*}, \ldots, x_{d}^{*}\right) \omega_{G}\right]_{d},
$$

and hence is zero by assumption. So in light of the inclusion $\Omega_{d-1} / \Omega_{d} \subset \omega_{G}$, the element $x$ must have been in $\Omega_{d}$ after all. The proof that the second condition implies the first is complete.

For the converse, consider a degree $d$ element in

$$
\bigcap_{\left\{x_{1}, \ldots, x_{d}\right\} \in \mathcal{R}}\left(x_{1}^{*}, \ldots, x_{d}^{*}\right) \omega_{G} .
$$

Our additional hypotheses imply that $\left[\omega_{G}\right]_{d} \cong \Omega_{d-1} / \Omega_{d}$, so this element is represented by some $x$ in $\Omega_{d-1}$. Likewise, since $\left[\omega_{G}\right]_{d-1} \cong\left(\Omega_{d-2} \cap \omega_{A}\right) / \Omega_{d-1}$ and $\Omega_{d} \subset J \omega_{A}$, we can assume with out loss of generality that

$$
x \in\left(x_{1}, \ldots, x_{d}\right)\left(\Omega_{d-2} \cap \omega_{A}\right),
$$

for any $\left(x_{1}, \ldots, x_{d}\right) \in \mathcal{R}$. Since such $x$ in is $\bigcap_{\left\{x_{1}, \ldots, x_{d}\right\} \in \mathcal{R}}\left(x_{1}, \ldots, x_{d}\right) \omega_{A}$, our hypothesis ensures that it is in $\Omega_{d}$, so that $x$ represents the zero class in $\omega_{G}$. This implies that

$$
\bigcap_{\left\{x_{1}, \ldots, x_{d}\right\} \in \mathcal{R}}\left[\left(x_{1}^{*}, \ldots, x_{d}^{*}\right) \omega_{G}\right]_{d}=0
$$


3.4. The Proof for $\omega_{G}$. In light of the Key Lemma 3.3.1 the next result will complete the proof of Theorem 3.0.2

Theorem 3.4.1. Let $(A, \mathfrak{m})$ be a local ring containing the rational numbers. Let $I$ be any $\mathfrak{m}$-primary ideal. Then

$$
\bigcap_{\left(x_{1}, \ldots, x_{d}\right) \in \mathcal{R}}\left[\left(x_{1}^{*}, \ldots, x_{d}^{*}\right) \omega_{G}\right]_{d}=0,
$$

where $\mathcal{R}$ is the set of all minimal reductions of $I$. Furthermore, if $A$ is the localization of a finitely generated $\mathbb{N}$-graded domain $S$ and $I$ is the expansion to $A$ of a homogeneous ideal of $S$ generated by elements of all the same degree $n$, then the set $\mathcal{R}$ can be taken to be the set of all reductions of I generated by elements of degree $n$.

We will prove Theorem 3.4.1 by induction on $d$. The hard part will be to deal with the case where $d=1$. The inductive step looks slightly technical also, because of the necessity of dealing with non-Cohen-Macaulay associated graded rings. We isolate most of these technicalities in the following lemma, which compares the canonical modules of two graded rings closely related to $G$. The reader is advised to think about the case where $G$ is Cohen-Macaulay on a first read through, as this simplifies the arguments and is sufficient for our main algebraic results (but not for Kawamata's Conjecture).

Lemma 3.4.2. Let $(A, \mathfrak{m})$ be a local ring of dimension $d \geq 2$, and let $G$ be the associated graded ring of $A$ with respect to an $\mathfrak{m}$-primary ideal $I$. Let $y \in I \backslash I^{2}$ be a general element of $I$, and let $y^{*}=y+I^{2}$ denote the corresponding degree one element in $G$. Then

(1) $(y) \cap I^{n}=y I^{n-1}$ for all $n \gg 0$.

(2) There is a natural degree preserving surjection

$$
G / y^{*} G \longrightarrow \bar{G}:=\frac{\bar{A}}{\bar{I}} \oplus \frac{\bar{I}}{\bar{I}^{2}} \oplus \frac{\bar{I}^{2}}{\bar{I}^{3}} \oplus \ldots,
$$

where $\bar{G}$ denotes the associated graded ring of $\bar{A}=A / y A$ with respect to the image ideal $\bar{I}=I \bar{A}$, which becomes an bijection in degrees $n \gg 0$.

(3) This surjection induces a degree preserving isomorphism

$$
\omega_{\bar{G}} \longrightarrow \omega_{G / y^{*} G}
$$

Remark 3.4.3. When the associated graded ring $G$ is Cohen-Macaulay (as it is in our main applications), the equality holds in (1) for all $n>0$ and the map in (2) is an isomorphism, making (3) obvious.

Proof. Condition (1) is easy to prove and well-known: the point is to choose $y$ so that $y^{*}$ avoids the relevant associated primes of $G$. (Such an element $y$ is called "filter regular.") See, for example, 44, Lemma 3.2].

Condition (2) follows immediately. Indeed, one simply verifies that in degree $n$, this map looks like

$$
\frac{I^{n}}{x I^{n-1}+I^{n+1}} \longrightarrow \frac{I^{n}}{(x) \cap I^{n}+I^{n+1}} .
$$

This is obviously surjective for all $n$, and becomes an isomorphism when $n \gg 0$. 
To prove (3), note that the kernel of the natural degree preserving surjection

$$
G / y^{*} G \longrightarrow \bar{G}
$$

is non-zero in only finitely many degrees, and therefore has finite length. In particular, the kernel $K$ is a zero dimensional graded $G / y^{*} G$-module. As both graded rings above have dimension $(d-1)$, the corresponding long exact sequence of local cohomology induces an isomorphism

$$
H_{\mathfrak{M}_{G / y^{*} G}}^{d-1}\left(G / y^{*} G\right) \longrightarrow H_{\mathfrak{M}_{\bar{G}}}^{d-1}(\bar{G})
$$

since $H_{\mathfrak{M}_{G}}^{d-1}(K)=0$.

Dually (after applying the graded Matlis dual; see Subsection [2.6), we have the desired degree preserving isomorphism

$$
\omega_{\bar{G}} \longrightarrow \omega_{G / y^{*} G}
$$

Proof of Theorem 3.4.1. We first carry out the inductive step. Assume that $d>1$ and that the theorem has been proven for rings $A$ of dimension $d-1$. Suppose that

$$
\omega \in \bigcap_{\left(x_{1}, \ldots, x_{d}\right) \in \mathcal{R}}\left[\left(x_{1}^{*}, \ldots, x_{d}^{*}\right) \omega_{G}\right]_{d} .
$$

We will construct a sequence $y_{1}, y_{2} \ldots$ of elements of $I$ (of degree $n$ in the graded case) such that the corresponding elements $y_{1}^{*}, y_{2}^{*} \ldots$ of $G_{1}$ are nonzerodivisors on $\omega_{G}$ with the property that

$$
\omega \in\left(\prod_{i=1}^{t} y_{i}^{*}\right) \omega_{G}
$$

for all $t \geq 1$. This will imply the claim, because then $\omega \in \omega_{G}$ would be divisible by elements of arbitrarily large degree, which is impossible since $\omega_{G}$ is Noetherian, and hence vanishes in sufficiently negative degrees.

To construct the sequence of $y_{i}$ 's, we proceed inductively. Assuming that $y_{1}, \ldots, y_{t-1}$ have already been constructed, choose a general $y_{t} \in I$ (of degree $n$ in the graded case) such that $\left\{y_{1}^{*} \cdots y_{t-1}^{*}, y_{t}^{*}\right\}$ is part of a sequence of parameters for $G$. This is possible, since the elements $x^{*}$ with $x \in I$ (of degree $n$ ) generate an $\mathfrak{M}_{G}$-primary ideal. We must verify that $\omega$ is divisible by the product $y_{1}^{*} \cdots y_{t-1}^{*} y_{t}^{*}$.

Consider the class of $\omega$ modulo $y_{t}^{*} \omega_{G}$. This is an element of $\omega_{G} / y_{t}^{*} \omega_{G}$ of degree $d$, so in light of the injection provided by Proposition [2.6.1] it determines an element of degree $d-1$ in $\omega_{\frac{G}{y_{t}^{G} G}}$. By Lemma 3.4.2 therefore, we can interpret it as an element in

$$
\left[\omega_{\bar{G}}\right]_{d-1},
$$

where $\bar{G}$ is the associated graded ring of the ring $A / y_{t} A$ with respect to the image of $I$.

Now any minimal reduction of $\bar{I}$ in $\bar{A}$, say $\left(\overline{x_{1}}, \ldots, \overline{x_{d-1}}\right)$, lifts to a minimal reduction $\left(x_{1}, \ldots, x_{d-1}, y_{t}\right)$ of $I$, because, again by Lemma 3.4 .2 for all $r \gg 0$,

$$
I^{r+1} \subset\left(x_{1}, \ldots, x_{d-1}\right) I^{r}+\left(y_{t}\right) \cap I^{r+1} \subset\left(x_{1}, \ldots, x_{d-1}\right) I^{r}+y_{t} I^{r} .
$$


It thus follows that

$$
\bar{\omega} \in \bigcap_{\overline{x_{1}}, \ldots, \overline{x_{d-1}}}\left[\left({\overline{x_{1}}}^{*}, \ldots,{\overline{x_{d-1}}}^{*}\right) \omega_{\bar{G}}\right]_{d-1}=0,
$$

where $\overline{x_{1}}, \ldots, \overline{x_{d-1}}$ ranges through the set of all minimal reductions of $\bar{I}$ in $\bar{A}$. (In the graded case, we assume the $x_{i}$ 's all to have degree $n$.)

By the inductive hypothesis, we can assume the result is true for the $(d-1)$ dimensional ring $\bar{G}$, which is the associated graded ring for an $\mathfrak{m}$-primary ideal in a ring of dimension $d-1$. So we have $\bar{\omega}=0$. In other words $\omega \in\left(y_{t}^{*}\right) \omega_{G}$. This means that $\omega \in\left(y_{1}^{*} \cdots y_{t-1}^{*}\right) \omega_{G} \cap\left(y_{t}^{*}\right) \omega_{G}$. Because the two element set $\left\{y_{1}^{*} \cdots y_{t-1}^{*}, y_{t}^{*}\right\}$ is an $\omega_{G}$-regular sequence, this means that $\omega \in\left(y_{1}^{*} \cdots y_{t}^{*}\right) \omega_{G}$ as wanted. This completes the proof of the inductive step.

It remains only to prove the case where $d=1$. For this, we will invoke the careful description of $\omega_{G}$ in the one dimensional case proved in Lemma 3.2.1] We will also need the following two lemmas. The first is a modification of [7] Lemma 2.2], which in turn is inspired by [22, Lemma 3.8]. The second is a one-dimensional version of [24 Proposition 2.3] valid also in the non-Cohen-Macaulay case.

Lemma 3.4.4. Let $(A, \mathfrak{m})$ be a local ring and let $N \subset N^{\prime} \subset M$ be A-modules. Let $x, y \in A$, with $x$ a nonzerodivisor on $M$. Suppose that $y N \subset x N$. Let $u_{1}, \ldots, u_{m} \in$ $A$ be units of $A$ whose images are distinct modulo $\mathfrak{m}$, and assume that $m \gg 0$ (specifically, $m>\operatorname{dim}_{k}\left(N:_{N^{\prime}} \mathfrak{m}\right) / N$, where $\left.k=A / \mathfrak{m}\right)$. Then

$$
x\left(N:_{M} \mathfrak{m}\right) \quad \bigcap \bigcap_{i=1}^{m}\left(x+u_{i} y\right)\left(N:_{N^{\prime}} \mathfrak{m}\right) \subset x N^{\prime} .
$$

Proof. Suppose that $w=x s=\left(x+u_{1} y\right) s_{1}=\ldots=\left(x+u_{m} y\right) s_{m}$ where $s \in N:_{M} \mathfrak{m}$ and $s_{i} \in\left(N:_{N^{\prime}} \mathfrak{m}\right)$ for each $i=1, \ldots, m$. For all $r \in N:_{M} \mathfrak{m}$, let $\bar{r}$ denote the class $r+N$ in $N:_{M} \mathfrak{m} / N$. Also let $\bar{a}$ denote the class $a+\mathfrak{m} \in A / \mathfrak{m}$ for all $a \in A$. The elements $\overline{u_{1}} \overline{s_{1}}, \ldots, \overline{u_{m}} \overline{s_{m}} \in\left(N:_{N^{\prime}} \mathfrak{m}\right) / N$ are linearly dependent, since $m$ exceeds the dimension of this space. By replacing $m$ by a possibly smaller positive integer, we will assume henceforth that every proper subset of the set

$$
\left\{\overline{u_{1}} \overline{s_{1}}, \ldots, \overline{u_{m}} \overline{s_{m}}\right\}
$$

is linearly independent, but that the full set itself is dependent. Clearly $m>1$; otherwise, all $s_{i}$ are in $N$ and so $\left(x+u_{i} y\right) s_{i} \in x N \subset x N^{\prime}$ as needed.

There exist units $\lambda, \ldots, \lambda_{m} \in A$ such that

$$
\sum_{i=1}^{m} \overline{\lambda_{i}} \overline{u_{i}} \overline{s_{i}}=0
$$

This implies

$$
\sum_{i=1}^{m} \lambda_{i} u_{i} s_{i} \in N, \quad \text { so that }\left(\sum_{i=1}^{m} \lambda_{i} u_{i} s_{i}\right) y \in y N \subset x N \text {. }
$$

Hence

$$
\left(\sum_{i=1}^{m} \lambda_{i} u_{i} s_{i}\right) y=t x
$$


for some $t \in N$. Set

$$
\lambda=\sum_{i=1}^{m} \lambda_{i}
$$

Then

$$
\lambda s x=\sum_{i=1}^{m} \lambda_{i} s_{i}\left(x+u_{i} y\right)=\sum_{i=1}^{m} \lambda_{i} s_{i} x+\sum_{i=1}^{m} \lambda_{i} s_{i} u_{i} y=\sum_{i=1}^{m} \lambda_{i} s_{i} x+t x .
$$

As $x$ is $M$-regular, this implies that

$$
\lambda s=\sum_{i=1}^{m} \lambda_{i} s_{i}+t .
$$

Now $\lambda \in \mathfrak{m}$ would imply $\lambda s \in N$ and so

$$
\sum_{i=1}^{m} \overline{\lambda_{i}} \overline{s_{i}}=0
$$

In this case, we can solve

$$
\bar{s}_{m}=-\sum_{i=1}^{m-1} \frac{\bar{\lambda}_{i}}{\bar{\lambda}_{m}} \bar{s}_{i}
$$

and plug into equation (8) above. Because the $\bar{u}_{i}$ 's are pairwise distinct, this produces a non-trivial linear relation on the set

$$
\left\{\bar{u}_{1} \bar{s}_{1}, \ldots, \bar{u}_{m} \bar{s}_{m-1}\right\},
$$

a contradiction. This forces $\lambda$ to be a unit in $A$, and so

$$
s=\sum_{i=1}^{m} \lambda^{-1} \lambda_{i} s_{i}+\lambda^{-1} t \in N^{\prime} .
$$

The proof of Lemma 3.4 .4 is complete.

Lemma 3.4.5. Let $I$ be an $\mathfrak{m}$-primary ideal in a local ring $(A, \mathfrak{m})$ of dimension one. Let $(x)$ be any minimal reduction of $I$. Then

$$
\Omega_{0}=\left(x^{t} \omega_{A}: \omega_{A} I^{t}\right)
$$

for all $t \gg 0$.

Proof. Note that the blowup of $\operatorname{Spec} A$ along $I$ is the affine scheme $Y=\operatorname{Spec} A\left[\frac{I}{x}\right]$. So the proper map $Y \longrightarrow \operatorname{Spec} A$ corresponds to a finite map of rings $A \rightarrow A\left[\frac{I}{x}\right]$. Thus

$$
\Omega_{0}=\omega_{\mathcal{O}_{Y}}=\operatorname{Hom}_{A}\left(\mathcal{O}_{Y}, \omega_{A}\right) .
$$

To compute $\Omega_{0}$, note that without loss of generality, the $A$-module generators for the ring $\mathcal{O}_{Y}$ may be assumed of the form $\frac{z}{x^{t}}$ where $z \in I^{t}$, for some fixed $t \gg 0$. We claim that $\Omega_{0}=\left(x^{t} \omega_{A}: \omega_{A} I^{t}\right)$ for this fixed $t$.

To check the inclusion $\Omega_{0} \subset\left(x^{t} \omega_{A}: \omega_{A} I^{t}\right)$, take any $f \in \operatorname{Hom}_{A}\left(\mathcal{O}_{Y}, \omega_{A}\right)$. Then the restriction of $f$ to $A$ is given by multiplication by $f(1) \in \omega_{A}$. Because $f\left(\frac{z}{x^{t}}\right) \in$ $\omega_{A}$ for any $z \in I^{t}$, one readily verifies that $f(z) \in x^{t} \omega_{A}$ and so $f(1) I^{t} \in\left(x^{t}\right) \omega_{A}$. Then the map $f \mapsto f(1)$ gives the natural inclusion $\Omega_{0} \subset\left(x^{t} \omega_{A}: \omega_{A} I^{t}\right)$.

For the reverse inclusion, take any $w \in\left(x^{t} \omega_{A}: \omega_{A} I^{t}\right)$. For $z \in I^{t}$, we have $w z=x^{t} u$ for some $u \in \omega_{A}$. Set $f_{w}\left(\frac{z}{x^{t}}\right)=u$. Using the fact that $x$ is a nonzerodivisor on $\omega_{A}$, one easily checks that the association $w \mapsto f_{w}$ gives a well-defined injection $\left(x^{t} \omega_{A}: \omega_{A} I^{t}\right) \subset \operatorname{Hom}_{A}\left(\mathcal{O}_{Y}, \omega_{A}\right)$ inverse to the map in the previous paragraph. 
We now prove the dimension one case of Theorem 3.4.1 which will complete its proof, and hence the proof of the main technical theorem, Theorem 3.0.2.

Let $d=1$. Suppose that $\omega \in\left[x^{*} \omega_{G}\right]_{1}$ for all $x \in I$ which generate a minimal reduction of $I$ (of degree $n$ in the graded case). As $x$ is not in any minimal prime of $A$, the element $x$ forms a system of parameters for $A$ and so $x$ is nonzerodivisor on the $A$-module $\omega_{A}$. By Lemma 3.2.1 we know that $\left[\omega_{G}\right]_{1}=\Omega_{0} / \Omega_{1}$. So we represent $\omega$ by some for some $w \in \Omega_{0}$, modulo $\Omega_{1}$. We want to prove that $\omega=0$, which is the same thing as proving that $w \in \Omega_{1}$.

Because the module $\left[\omega_{G}\right]_{1}=\Omega_{0} / \Omega_{1}$ is an Artinian $A$-module, we might as well assume that $\mathfrak{m} \omega=0$. In other words, we can assume that $w \in \Omega_{1}:_{\Omega_{0}} \mathfrak{m}$. Again by Lemma 3.2.1] we know that $\left[\omega_{G}\right]_{0}=\left(\Omega_{-1} \cap \omega_{A}\right) / \Omega_{0}$. So, using also that $\Omega_{1}=x \Omega_{0}$ by Lemma 3.1.4 we can write $w=s x$ for some $s \in \Omega_{-1} \cap \omega_{A}$. Since

$$
\mathfrak{m} s x=\mathfrak{m} w \in \Omega_{1}=x \Omega_{0},
$$

we have that

$$
\mathfrak{m} s \in \Omega_{0},
$$

as $x$ is a nonzerodivisor on $\omega_{A}$. This means that $s \in \Omega_{0}: \omega_{A} \mathfrak{m}$. Our goal is to show $s \in \Omega_{0}$. This will complete the proof since then $w=x s \in I \Omega_{0} \subset \Omega_{1}$, whence the class of $w$ in $\omega_{G}$ is zero.

To achieve our goal of showing that $s \in \Omega_{0}$, we invoke Lemma 3.4.5 which guarantees that it is enough to show that $s \in x^{t} \omega_{A}: I^{t}$ for $t \gg 0$. For this, note that it is enough to show that

Claim 3.4.6. For any $y \in I$ (homogeneous in the graded case), $s \in\left(x^{t} \omega_{A}: \omega_{A} y^{t}\right)$ for all $t \gg 0$.

Indeed, because $\mathbb{Q} \subset A$, the ideal generated by the $t$-th powers of the elements of an ideal $I$ (homogeneous elements of degree $n$ when $I$ is generated in degree $n$ ) is simply $I^{t}$. This follows easily from the identity

$$
t ! X_{1} \cdots X_{t}=\sum_{1 \leq k \leq t} \sum_{1 \leq i_{1}<\ldots<i_{k} \leq t}(-1)^{t-k}\left(X_{i_{1}}+\ldots+X_{i_{k}}\right)^{t} .
$$

To prove Claim 3.4.6 take any $y \in I$ (of degree $n$ in the graded case). We wish to apply Lemma 3.4 .4 to the $A$-modules

$$
\Omega_{0} \subset N_{t}^{\prime} \subset \omega_{A},
$$

where $N_{t}^{\prime}=\left(x^{t} \omega_{A}: \omega_{A} y^{t}\right)$. Note here that $\Omega_{0} \subset N_{t}^{\prime}$ for all $t$, since

$$
y^{t} \Omega_{0} \subset I^{t} \Omega_{0} \subset \Omega_{t}=x^{t} \Omega_{0} \subset x^{t} \omega_{A} .
$$

To this end, choose distinct units $u_{1}, \ldots, u_{m}$ (of degree zero in the graded case), with $m \gg 0$, and so that each $x+u_{i} y$ generates a reduction for $I$. Then

$$
w=x s=\left(x+u_{1} y\right) s_{1}=\ldots=\left(x+u_{m} y\right) s_{m}
$$

for some $s_{1}, \ldots, s_{m} \in \Omega_{0}: \omega_{A} \mathfrak{m}$. Therefore, assuming for the moment that the elements $s_{i}$ are also in $N_{t}^{\prime}$, then $x s$ is in

$$
x\left(\Omega_{0}: \omega_{A} \mathfrak{m}\right) \cap\left[\left(x+u_{1} y\right)\left(\Omega_{0}:_{N_{t}^{\prime}} \mathfrak{m}\right)\right] \cap \cdots \cap\left[\left(x+u_{m} y\right)\left(\Omega_{0}:_{N_{t}^{\prime}} \mathfrak{m}\right)\right],
$$

and so applying Lemma 3.4.4 we have

$$
x s \in x N_{t}^{\prime} \text {. }
$$


Because $x$ is a nonzerodivisor on $\omega_{A}$ (and so on any submodule), we see that $s \in N_{t}^{\prime}=\left(x^{t} \omega_{A}: \omega_{A} y^{t}\right)$, which is precisely what we needed to show.

It remains to show that each $s_{i}$ is in $N_{t}^{\prime}=\left(x^{t} \omega_{A}: \omega_{A} y^{t}\right)$ for $t \gg 0$. We in fact will show this for all $t \geq 0$, using induction on $t$. If $t=0$, the $s_{i}$ are trivially in $N_{0}^{\prime}$. Assume then that

$$
s_{1}, \ldots, s_{m} \in N_{t-1}^{\prime}=\left(x^{t-1} \omega_{A}:_{\omega_{A}} y^{t-1}\right) .
$$

So by the argument above using Lemma 3.4.4 we have also that $s \in N_{t-1}^{\prime}$. Since

$$
x s=\left(x+u_{i} y\right) s_{i},
$$

we have

$$
y s_{i}=u_{i}^{-1} x\left(s-s_{i}\right) \in x N_{t-1}^{\prime}=x\left(x^{t-1} \omega_{A}:_{\omega_{A}} y^{t-1}\right) \subset x^{t} \omega_{A}:_{\omega_{A}} y^{t-1} .
$$

But then for each $i=1, \ldots, m$, we have

$$
s_{i} \in\left(x^{t} \omega_{A}: \omega_{A} y^{t}\right)=N_{t}^{\prime},
$$

as we sought to show.

This completes the proof of Claim [3.4.6] and hence the proof of Theorem 3.4.1 in the dimension one case, and the proof of our main technical theorem, Theorem 3.0 .2

Remark 3.4.7. In Theorem 3.0.2 as well as in its later applications in Section 5, the assumption that $A$ contains the set of rational numbers is unnecessary in case the ideal $I$ has reduction number at most one. Note that the reduction number

does not increase as we reduce to the one-dimensional case. Now it is easy to see that in Lemma 3.4.5 one can take $t$ to be the reduction number, that is, $\Omega_{0}=\left(x^{t} \omega_{A}: \omega_{A} I^{t}\right)=\left(x^{r} \omega_{A}: \omega_{A} I^{r}\right)$ where $r$ is the reduction number of $I$. So when the reduction number of $I$ is at most one, it suffices to prove Claim 3.4.6 for the case $t=1$. This follows in the same way without making use of Identity (9).

\section{Briançon-Skoda Type Results.}

In this section, we identify general conditions under which the hypothesis of our Main Technical Theorem 3.0.2 are satisfied. The results of this section will allow us to apply the main technical theorem proved in the preceding section to deduce our main results in the following section.

4.1. The Briançon-Skoda Theorem and related properties of adjoints. The next result is analogous to the "Briançon-Skoda Theorem with adjoints" proved by Lipman for regular schemes in [35. See also the "Skoda Theorem" discussed in 31.

Lemma 4.1.1. Let $(A, \mathfrak{m})$ be a local ring of dimension $d \geq 1$, and let $I \subset A$ be an ideal of positive height. Assume that $\omega_{Y}$ is m-regular in the sense of CastelnuovoMumford, meaning that

$$
H^{i}\left(Y, \omega_{Y}(m-i)\right)=0
$$

for all $i>0$. Then $\Omega_{n+1}=J \Omega_{n}$ for all $n \geq m$, where $J$ is any reduction of $I$. 
Proof. This follows from a standard argument. As $I^{r+1}=J I^{r}$, we have $J \mathcal{O}_{Y}=$ $I \mathcal{O}_{Y}=\mathcal{O}_{Y}(1)$. Generators of $J$ therefore give rise to global sections which generate the sheaf $\mathcal{O}_{Y}(1)$. These give rise to an exact Koszul complex. One can then argue as in [35. p. 747] (or as below in the proof of Proposition 4.1.4) to conclude that $\Omega_{n+1}=J \Omega_{n}$ for all $n \geq m$ under the stated vanishing conditions.

Corollary 4.1.2. If $I$ is $\mathfrak{m}$-primary, and $A$ and $R=A[I t]$ are Cohen-Macaulay, then

$$
\Omega_{n}=J \Omega_{n-1}
$$

for all $n \geq d=\operatorname{dim} A$.

Proof. By Proposition 4.1.1 it suffices to check that $\omega_{Y}$ is $(d-1)$ regular. But the the vanishing of $H^{i}\left(Y, \omega_{Y}(d-1-i)\right)$ for positive $i$ is the same as the vanishing of $H_{Z}^{i}\left(Y, \mathcal{O}_{Y}(-i+1)\right)$ for $i \leq d-1$. This vanishing follows easily from the Sancho de Salas sequence for $R$, as recorded in 2.5.2

Remark 4.1.3. Corollary 4.1.1 holds also for ideals $I$ that have a reduction generated by regular sequence, with $d$ now the height of $I$. Furthermore, because $I \mathcal{O}_{Y}=$ $\mathcal{O}_{Y}(1)$ is ample for the map $\pi$, we have $H^{i}\left(Y, \omega_{Y}(m-i)\right)=0$ for all $i>0$ and for sufficiently large $m$. So for large enough $n$, we always have $\Omega_{n+1}=J \Omega_{n}$, where $J$ is any reduction of $I$.

The next result ensures that Theorem 3.0 .2 can be applied to some interesting cases. Note that when $n \geq d$, this statement collapses to the Briançon-Skoda Theorem above in 4.1.2 the following result thus informs us also of what goes on for smaller indices $n$.

Proposition 4.1.4. Let $I$ be an $\mathfrak{m}$-primary ideal in a Cohen-Macaulay ring $(A, \mathfrak{m})$ of dimension $d>0$, and let $J$ be any minimal reduction of $I$. Let $R$ denote the Rees ring $A[I t]$ of $A$ with respect to $I$, and assume that $R$ is Cohen-Macaulay. Then

$$
J \omega_{A} \cap \Omega_{n}=J \Omega_{n-1}
$$

for all $n \in \mathbb{Z}$.

Proof. The case $d=1$ is degenerate. The condition that $R$ is Cohen-Macaulay forces $I$ to be principle (see e. g. [19, Cor 25.2]), so $I=J=(x)$. Then $Y=\operatorname{Spec} A$ and $\Omega_{n}=x^{n} \omega_{A}$ for all $n$. This makes the statement obvious.

Assume $d>1$. Because $A$ and $R$ are both Cohen-Macaulay, $\Omega_{n}=\omega_{A}$ for nonpositive $n$; see Lemma 3.1.3 and 2.5.2 So the statement is trivial for negative $n$. Also, when $n \geq d$, the statement follows from Corollary 4.1.2 It remains to consider the case $1 \leq n<d$.

Let us first consider the weaker statement that

$$
J \Omega_{n-2} \cap \Omega_{n}=J \Omega_{n-1}
$$

for all $n<d$. To prove this, it is sufficient to prove that the natural map

$$
\Omega_{n} / J \Omega_{n-1} \longrightarrow \Omega_{n-1} / J \Omega_{n-2}
$$

is injective. 
Because $I^{r+1}=J I^{r}$ for some $r \geq 0$, we have $I \mathcal{O}_{Y}=J \mathcal{O}_{Y}$. Fix generators $\left\{x_{1}, \ldots, x_{d}\right\}$ for $J$ as an ideal of $A$. These elements give rise to generating global sections of $I \mathcal{O}_{Y}$, and hence a surjection $\bigoplus_{i=1}^{d} \mathcal{O}_{Y} \longrightarrow I \mathcal{O}_{Y}$, and so also a surjection

$$
\mathcal{F}:=\bigoplus_{i=1}^{d} \mathcal{O}_{Y}(-1) \stackrel{\left(\begin{array}{c}
x_{1} \\
\vdots \\
x_{d}
\end{array}\right)}{\longrightarrow} \mathcal{O}_{Y} .
$$

This means that the Koszul complex

$$
0 \longrightarrow \wedge^{d} \mathcal{F} \otimes \omega_{Y}(n) \longrightarrow \ldots \longrightarrow \wedge^{1} \mathcal{F} \otimes \omega_{Y}(n) \longrightarrow \omega_{Y}(n) \longrightarrow 0
$$

is exact, where

$$
\wedge^{j} \mathcal{F} \otimes \omega_{Y}(n)=\left(\omega_{Y}(n-j)\right)^{\oplus\left(\begin{array}{l}
d \\
j
\end{array}\right)},
$$

for all $j=0,1, \ldots, d$. We now split this complex into $d-1$ short exact sequences

$$
0 \longrightarrow \mathcal{K}_{j} \longrightarrow \wedge^{j} \mathcal{F} \otimes \omega_{Y}(n) \longrightarrow \mathcal{K}_{j-1} \longrightarrow 0,
$$

where $\mathcal{K}_{0}=\omega_{Y}(n)$ and $\mathcal{K}_{d-1}=\omega_{Y}(n-d)$. The corresponding long exact sequences of cohomology give the exact sequences

$$
H^{j-1}\left(Y, \wedge^{j} \mathcal{F} \otimes \omega_{Y}(n)\right) \longrightarrow H^{j-1}\left(Y, \mathcal{K}_{j-1}\right) \longrightarrow H^{j}\left(Y, \mathcal{K}_{j}\right)
$$

for each $j=1,2, \ldots, d-1$. When $j=1$ we get the sequence

$$
\bigoplus_{i=1}^{d} \Omega_{n-1} \stackrel{\left(\begin{array}{c}
x_{1} \\
\vdots \\
x_{d}
\end{array}\right)}{\longrightarrow} \Omega_{n} \longrightarrow H^{1}\left(Y, \mathcal{K}_{1}\right)
$$

which gives an injection

$$
\Omega_{n} / J \Omega_{n-1} \hookrightarrow H^{1}\left(Y, \mathcal{K}_{1}\right) .
$$

Note that $H^{j-1}\left(Y, \omega_{Y}(n-j)\right)=0$ for $1<j \leq d-1$; this follows easily from the Sancho de Salas sequence (see 2.5.2), taking into consideration the abundant vanishing afforded because $A$ and $R$ are Cohen-Macaulay. Thus we obtain injections

$$
H^{j-1}\left(Y, \mathcal{K}_{j-1}\right) \hookrightarrow H^{j}\left(Y, \mathcal{K}_{j}\right)
$$

and finally, an injection

$$
\Omega_{n} / J \Omega_{n-1} \hookrightarrow H^{d-1}\left(Y, \omega_{Y}(n-d)\right) .
$$

The inclusion $I^{n} \omega_{Y} \subset I^{n-1} \omega_{Y}$ induces a homomorphism of complexes

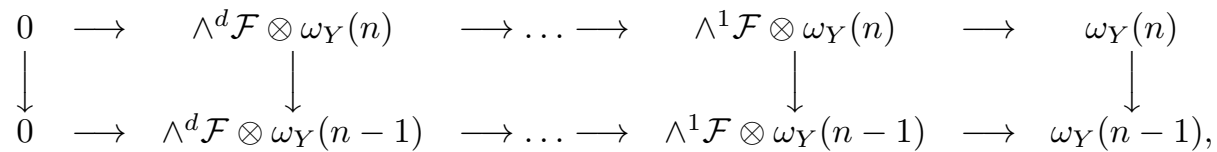

which in turn induces a commutative diagram

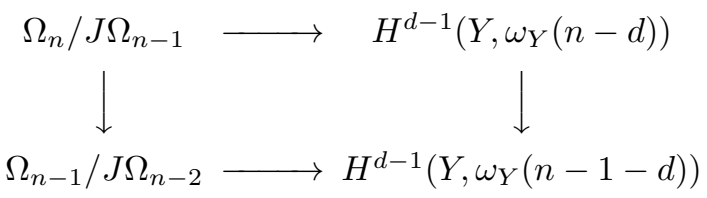


Thus we can get the desired injectivity of $\Omega_{n} / J \Omega_{n-1} \longrightarrow \Omega_{n-1} / J \Omega_{n-2}$ by proving injectivity for the homomorphism

$$
H^{d-1}\left(Y, \omega_{Y}(n-d)\right) \longrightarrow H^{d-1}\left(Y, \omega_{Y}(n-1-d)\right) .
$$

To this end, consider the adjunction sequence for $E \subset Y$,

$$
0 \longrightarrow \omega_{Y} \longrightarrow \omega_{Y}(-1) \longrightarrow \omega_{E} \longrightarrow 0 \text {. }
$$

Tensor with the flat module $I^{n-d} \mathcal{O}_{Y}$ to get a short exact sequence

$$
0 \longrightarrow \omega_{Y}(n-d) \longrightarrow \omega_{Y}(n-1-d) \longrightarrow \omega_{E}(n-d) \longrightarrow 0 .
$$

Taking cohomology, we get the exact sequence

$$
H^{d-2}\left(E, \omega_{E}(n-d)\right) \longrightarrow H^{d-1}\left(Y, \omega_{Y}(n-d)\right) \longrightarrow H^{d-1}\left(Y, \omega_{Y}(n-1-d)\right) .
$$

Thus it is sufficient to show that $H^{d-2}\left(E, \omega_{E}(n-d)\right)=0$. By duality applied to the map $E \longrightarrow$ Spec $A / I$, it is enough to show $H^{1}\left(E, \mathcal{O}_{E}(d-n)\right)=0$. But this follows easily from the Sancho de Salas sequence for the graded ring $G$ :

$$
H_{\mathfrak{m}}^{1}\left(G_{d-n}\right) \longrightarrow H^{1}\left(E, \mathcal{O}_{E}(d-n)\right) \longrightarrow\left[H_{\mathfrak{M}_{G}}^{2}(G)\right]_{d-n} .
$$

Indeed, because $n<d$, the module $H_{\mathfrak{m}}^{1}\left(G_{d-n}\right)=H_{m}^{1}\left(I^{d-n} / I^{d-n+1}\right)$ vanishes (as the module $I^{d-n} / I^{d-n+1}$ has zero-dimensional support). Now when both $R$ and $A$ are Cohen-Macaulay, the associated graded $\operatorname{ring} G$ is Cohen-Macaulay with negative $a$-invariant (see 2.5.2), so the module $\left[H_{\mathfrak{M}_{G}}^{2}(G)\right]_{d-n}$ certainly vanishes, as $d-n>0$.

We have now shown that

$$
J \Omega_{n-2} \cap \Omega_{n}=J \Omega_{n-1}
$$

for all $n<d$. To complete the proof, note $J \Omega_{n-1} \subset J \omega_{A} \cap \Omega_{n}$ for all $n \geq 1$. So we need to show the reverse inclusion. We will do this by induction on $n$, starting from $n=0$.

For $n=0$, we verify that $J \omega_{A} \cap \Omega_{0} \subset J \Omega_{-1}$. Indeed, because the $a$-invariant of $G$ is non-positive, we know that $\omega_{A} \subset \Omega_{-1}$. So $J \omega_{A} \subset J \Omega_{-1}$, and of course then $J \omega_{A} \cap \Omega_{0} \subset J \Omega_{-1}$.

Now assume that $n>0$ and the inclusion has been proved for smaller indices. Take $x \in J \omega_{A} \cap \Omega_{n}$. Then certainly $x \in J \omega_{A} \cap \Omega_{n-1}$, which is $J \Omega_{n-2}$ by the induction hypothesis. So $x \in J \Omega_{n-2} \cap \Omega_{n}$. But by the weaker statement proved above, this implies that $x \in J \Omega_{n-1}$. The proof is complete.

4.2. The case where $A$ is not necessarily Cohen-Macaulay. The following proposition offers an even more general setting in which the hypothesis of our main technical theorem are satisfied. Its proof is decidedly less elementary than the argument we have already made for Proposition 4.1.4 (which is why we have included a separate proof for 4.1.4).

Proposition 4.2.1. Let $(A, \mathfrak{m})$ be a local ring of dimension $d>1$. Let $I \subset A$ be an $\mathfrak{m}$-primary ideal such that the irrelevant ideal of the Rees ring $A[I t]$ is a Cohen-Macaulay $A[I t]$-module. Then, for any reduction $J$ of $I$,

$$
J \omega_{A} \cap \Omega_{n}=J \Omega_{n-1}
$$

for all $n \in \mathbb{Z}$. 
Proof. Let $R^{+}$denote the irrelevant ideal of $R=A[I t]$. Because $R^{+}$is CohenMacaulay, the $a$-invariant of the associated graded $\operatorname{ring} G$ is non-positive, as one checks by looking at the Sancho de Salas sequence. So from Lemma 3.1.3 we have that $\Omega_{n}=\omega_{A}$ for $n<0$. Thus statement is trivial for $n \leq-1$. Also the case $n=0$ is clear, since $J \omega_{A} \cap \Omega_{0}=J \Omega_{-1} \cap \Omega_{0}=J \Omega_{-1}$. For $n>0$ we proceed by induction on $n$ as in the proof of Proposition 4.1.4 As in that argument, it is sufficient to prove the weaker statement that

$$
J \Omega_{n-2} \cap \Omega_{n}=J \Omega_{n-1}
$$

for all $n \geq 1$.

Choose $N \gg 0$ such that $\Omega_{N+1}=J \Omega_{N}$. Write

$$
l_{A}\left(\Omega_{-1} / \Omega_{N+1}\right)=l_{A}\left(\Omega_{-1} / \Omega_{0}\right)+\sum_{n=0}^{N} l_{A}\left(\Omega_{n} / \Omega_{n+1}\right),
$$

where $l_{A}(M)$ denotes the length of an $A$-module $M$, and

$$
l_{A}\left(\Omega_{-1} / J \Omega_{N}\right)=l_{A}\left(\Omega_{-1} / J \Omega_{-1}\right)+\sum_{n=0}^{N} l_{A}\left(J \Omega_{n-1} / J \Omega_{n}\right) .
$$

Then

$$
l_{A}\left(\Omega_{-1} / J \Omega_{-1}\right)=l_{A}\left(\Omega_{-1} / \Omega_{0}\right)+\sum_{n=0}^{\infty}\left(l_{A}\left(\Omega_{n} / \Omega_{n+1}\right)-l_{A}\left(J \Omega_{n-1} / J \Omega_{n}\right)\right) .
$$

Consider the $G$-module $W=\bigoplus_{n \geq 0} \Omega_{n-1} / \Omega_{n}$. Fix generators $\left\{x_{1}, \ldots, x_{d}\right\}$ for $J$ as an ideal of $A$. Let $J^{*}$ denote the ideal $\left(x_{1}^{*}, \ldots, x_{d}^{*}\right) \subset G$. Because

$$
\begin{aligned}
l_{G}\left(W / J^{*}\right) & =l_{A}\left(\Omega_{-1} / \Omega_{0}\right)+\sum_{n=1}^{\infty} l_{A}\left(\Omega_{n-1} / J \Omega_{n-2}+\Omega_{n}\right) \\
& =l_{A}\left(\Omega_{-1} / \Omega_{0}\right)+\sum_{n=1}^{\infty}\left(l_{A}\left(\Omega_{n-1} / \Omega_{n}\right)-l_{A}\left(J \Omega_{n-2}+\Omega_{n} / \Omega_{n}\right)\right) \\
& =l_{A}\left(\Omega_{-1} / \Omega_{0}\right)+\sum_{n=1}^{\infty}\left(l_{A}\left(\Omega_{n-1} / \Omega_{n}\right)-l_{A}\left(J \Omega_{n-2} / J \Omega_{n-2} \cap \Omega_{n}\right)\right) \\
& =l_{A}\left(\Omega_{-1} / \Omega_{0}\right)+\sum_{n=0}^{\infty}\left(l_{A}\left(\Omega_{n} / \Omega_{n+1}\right)-l_{A}\left(J \Omega_{n-1} / J \Omega_{n-1} \cap \Omega_{n+1}\right)\right),
\end{aligned}
$$

we now obtain

$$
\begin{aligned}
l_{G}\left(W / J^{*}\right)-l_{A}\left(\Omega_{-1} / J \Omega_{-1}\right) & \left.=\sum_{n=0}^{\infty}\left(l_{A}\left(J \Omega_{n-1} / J \Omega_{n}\right)\right)-l_{A}\left(J \Omega_{n-1} / J \Omega_{n-1} \cap \Omega_{n+1}\right)\right) \\
& =\sum_{n=0}^{\infty} l_{A}\left(J \Omega_{n-1} \cap \Omega_{n+1} / J \Omega_{n}\right) .
\end{aligned}
$$

In order to prove our claim we thus have to show that $l_{G}\left(W / J^{*}\right)-l_{A}\left(\Omega_{-1} / J \Omega_{-1}\right)=$ 0 . For this, recall the notion of the $\mathbb{I}$-invariant of a graded module (see e. g. 43 , p. 6]). Let $B$ be a graded ring defined over a local ring, and let $N$ be a graded 
$B$-module with $r=\operatorname{dim} N$. Then the $\mathbb{I}$-invariant

$$
\mathbb{I}(N)=\sum_{i=0}^{r-1}\left(\begin{array}{c}
r-1 \\
i
\end{array}\right) l_{B}\left(H_{\mathfrak{N}}^{i}(N)\right)
$$

where $\mathfrak{N}$ denotes the homogeneous maximal ideal of $B$. It is a general fact that if $\left(y_{1}, \ldots, y_{r}\right)$ is any homogeneous system of parameters for $B / \operatorname{Ann} N$, then always $l_{A}\left(N /\left(y_{1}, \ldots, y_{r}\right) N\right)-e\left(y_{1}, \ldots, y_{r} ; N\right) \leq \mathbb{I}(N)$, where $e$ denotes the multiplicity of $N$ with respect to $\left(y_{1}, \ldots, y_{r}\right)$. If the equality holds, then the system of parameters $\left(y_{1}, \ldots, y_{r}\right)$ is called standard.

By definition the multiplicity $e\left(J^{*} ; W\right)=e\left(G^{+} ; W\right)$ is equal to $\mathrm{d}$ ! times the leading coefficient of the numerical polynomial $l_{A}\left(W_{n}\right)$ where $n \gg 0$. But for $n \gg 0$,

$$
W_{n}=\Omega_{n-1} / \Omega_{n}=J^{n-1-N} \Omega_{N} / J^{n-N} \Omega_{N}
$$

showing that $e\left(J^{*} ; W\right)=e\left(J, \Omega_{N}\right)$. For any $\mathfrak{p} \in \operatorname{Spec} A, \mathfrak{p} \not \supset I$, we clearly have $\left(\Omega_{n}\right)_{\mathfrak{p}}=\omega_{A_{p}}$ for all $n \in \mathbb{Z}$. Therefore

$$
\begin{aligned}
e\left(J, \Omega_{N}\right) & =\sum_{\mathfrak{p} \in \operatorname{Min}} \sum_{, \operatorname{dim} A / \mathfrak{p}=d} l_{A_{\mathfrak{p}}}\left(\left(\Omega_{N}\right)_{\mathfrak{p}}\right) e(J+\mathfrak{p} / \mathfrak{p} ; A / \mathfrak{p}) \\
& =\sum_{\mathfrak{p} \in \operatorname{Min} A, \operatorname{dim} A / \mathfrak{p}=d} l_{A_{\mathfrak{p}}}\left(\omega_{A_{\mathfrak{p}}}\right) e(J+\mathfrak{p} / \mathfrak{p} ; A / \mathfrak{p}) \\
& =\sum_{\mathfrak{p} \in \operatorname{Min} A, \operatorname{dim} A / \mathfrak{p}=d} l_{A_{\mathfrak{p}}}\left(\left(\Omega_{-1}\right)_{\mathfrak{p}}\right) e(J+\mathfrak{p} / \mathfrak{p} ; A / \mathfrak{p}) \\
& =e\left(J ; \Omega_{-1}\right) .
\end{aligned}
$$

So $e\left(J^{*} ; W\right)=e\left(J ; \Omega_{-1}\right)$.

Set $\Omega=\bigoplus_{n>-1} \Omega_{n}$ and $\Omega^{\prime}=\bigoplus_{n>0} \Omega_{n}$. Observe that $\Omega^{\prime}=\omega_{R^{+}}$where $\omega_{R^{+}}$ denotes the canonical module of the $R$-module $R^{+}$, that is, $\Omega^{\prime}$ is the graded dual of the top local cohomology module of $R^{+}$with supports in the unique homogeneous maximal ideal. Indeed, a look at the Sancho de Salas sequence

$$
0 \longrightarrow H_{\mathfrak{m}}^{d}\left(R_{n}^{+}\right) \longrightarrow H_{E}^{d}\left(X, \mathcal{O}_{X}(n)\right) \longrightarrow\left[H^{d+1}\left(R^{+}\right)\right]_{n} \longrightarrow 0
$$

shows that there is an isomorphism $H_{E}^{d}\left(X, \mathcal{O}_{X}(n)\right)=\left[H^{d+1}\left(R^{+}\right)\right]_{n}$, for all $n \leq 0$. Dualizing, we then see that $\Omega_{n}=\left[\omega_{R^{+}}\right]_{n}$ for all $n \geq 0$. On the other hand, by considering the long exact sequence of cohomology corresponding to the exact sequence

$$
0 \longrightarrow R^{+} \longrightarrow R \longrightarrow A \longrightarrow 0
$$

and taking into account that $a(R)=-1$, we get $\left[H^{d+1}\left(R^{+}\right)\right]_{n}=\left[H^{d+1}(R)\right]_{n}=$ 0 for $n>0$ so that $\left[\omega_{R^{+}}\right]_{n}=0$ when $n<0$. By 39] Satz 3.2.2], it follows that $\Omega^{\prime}$ is Cohen-Macaulay. By means of the long exact sequences of cohomology corresponding to the exact sequences

$$
0 \longrightarrow \Omega^{\prime} \longrightarrow \Omega \longrightarrow \Omega_{-1}(1) \longrightarrow 0 \text { and } 0 \longrightarrow \Omega^{\prime} \longrightarrow \Omega(-1) \longrightarrow W \longrightarrow 0
$$

one then easily checks that $H_{\mathfrak{M}}^{i}(W)=H_{\mathfrak{m}}^{i}\left(\Omega_{-1}\right)$ for all $0 \leq i<d$. Therefore $\mathbb{I}(W)=$ $\mathbb{I}\left(\Omega_{-1}\right)$. As $a(G) \leq 0$, Lemma 3.1 .3 implies that $\Omega_{-1}=\omega_{A}$. Using 15, Theorem 1.1, Appendix] and [15. Corollary 6.18] we know that $\left(x_{1}, \ldots, x_{d}\right)$ is a standard 
system of parameters for $A$. Moreover, by [15] Theorem 3.17], then $\left(x_{1}, \ldots, x_{d}\right)$ is standard also for $\omega_{A}$. This implies that $\mathbb{I}\left(\omega_{A}\right)=l_{A}\left(\omega_{A} / J \omega_{A}\right)-e\left(J ; \omega_{A}\right)$. Therefore

$$
\begin{aligned}
l_{G}\left(W / J^{*}\right)-l_{A}\left(\Omega_{-1} / J \Omega_{-1}\right) & =\left(l_{G}\left(W / J^{*}\right)-e\left(J^{*} ; W\right)\right) \\
& -\left(l_{A}\left(\Omega_{-1} / J \Omega_{-1}\right)-e\left(J ; \Omega_{-1}\right)\right) \\
& \leq \mathbb{I}(W)-\mathbb{I}\left(\omega_{A}\right)=0
\end{aligned}
$$

as wanted. The proof is complete.

\section{The main local algebraic Results}

In this section, we prove our main results about the core in a local ring, including the theorems relating core, adjoint, and coefficients ideals, the "local" version of Kawamata's Conjecture, and a formula for core conjectured in [7. All are deduced from the main technical theorem, Theorem 3.0.2 using the Briançon-Skoda results of Section 4. Further corollaries for graded rings appear at the end of Section 6 .

5.1. Formulas for Core in the Cohen-Macaulay case. Recall that an equimultiple ideal in a local ring $(A, \mathfrak{m})$ is an ideal whose height equals its analytic spread (see [19, p58]). When the ring $A$ has an infinite residue field, an equimultiple ideal is precisely an ideal having a reduction generated by part of a system of parameters. Every $\mathfrak{m}$-primary ideal in a local ring is equimultiple.

Corollary 5.1.1. Let $(A, \mathfrak{m})$ be a Cohen-Macaulay local ring containing a field of characteristic zero, and let $I$ be an equimultiple ideal of height $h$ whose Rees ring $A[I t]$ is Cohen-Macaulay. Then

$$
\operatorname{core}(I)=H^{0}\left(Y, I^{h} \omega_{Y}\right):_{A} \omega_{A}=J^{r+1}:_{A} I^{r}
$$

where, as always, $H^{0}\left(Y, I^{h} \omega_{Y}\right)$ is considered as a submodule of $\omega_{A}$ via the trace map, and $J$ is any reduction of $I$.

Remark 5.1.2. In Corollary 5.1.1 if the height $h$ is two (or less), then the assumption that $A$ contains the rational numbers is not needed. See Remark 3.4.7 The case where $h$ is height one is trivial, because our assumption on the Rees ring forces $I$ to be principal.

Remark 5.1.3. The formula core $(I)=J^{r+1}: I^{r}$ is conjectured in 7, Conjecture 5.1] under more general hypothesis.

Corollary 5.1 .1 follows easily from the following theorem, which generalizes our main technical theorem to ideals that may not be $\mathfrak{m}$-primary.

Theorem 5.1.4. Let $(A, \mathfrak{m})$ be a Cohen-Macaulay local ring containing the set of rational numbers. Let $I \subset A$ be an equimultiple ideal of positive height $h$ such that the corresponding Rees ring $A[I t]$ is Cohen-Macaulay. Then

$$
\operatorname{core}\left(I \omega_{A}\right)=\Gamma\left(Y, I^{h} \omega_{Y}\right)
$$

as submodules of $\omega_{A}$, where $Y=\operatorname{Proj} A[I t]$.

Remark 5.1.5. In fact, $\Omega_{h} \subset$ core $\left(I \omega_{A}\right)$ for equimultiple ideals $I$ in a CohenMacaulay local ring $A$ without any assumption on the Rees ring. The point is to prove the reverse inclusion. As we'll see in the proof, the reverse inclusion holds even when it is assumed only that the irrelevant ideal of $R$ is Cohen-Macaulay (even when $A$ is not). 
To see that that Theorem [5.1.4 implies Corollary 5.1.1] we need the following lemma.

Lemma 5.1.6. Let $(A, \mathfrak{m})$ be a Cohen-Macaulay local ring, and let $I$ be an equimultiple ideal of height $h$. Let $J$ be any minimal reduction of $I$. Then

$$
\Omega_{h}:_{A} \omega_{A}=J^{r+1}:_{A} I^{r}
$$

for any integer $r$ such that $I^{r+1}=J I^{r}$. In particular, $J^{r+1}: I^{r}$ is independent of the choice of reduction $J$.

Proof. We first note that

$$
\Omega_{n}=J^{n-h+r+1} \omega_{A}: \omega_{A} I^{r}
$$

for all $n \geq 0$. Indeed, set $X=\operatorname{Proj} A[J t]$ and $Y=\operatorname{Proj} A[I t]$. Since $J$ is generated by a regular sequence, $\omega_{A[J t]}$ has the expected form:

$$
\left[\omega_{A[J t}\right]_{n}=\Gamma\left(X, J^{n} \omega_{X}\right)=J^{n-h+1} \omega_{A}
$$

for all $n \geq 1$ (see e.g. [45, p. 142]). The canonical sheaf for $Y$ can therefore be computed from the canonical sheaf for $X$ via the finite map $Y \longrightarrow X$ induced by the inclusion of the Rees rings $A[J t] \hookrightarrow A[I t]$. Working this out, we arrive at

$$
\Gamma\left(Y, I^{n} \omega_{Y}\right)=\Gamma\left(X, J^{n+r} \omega_{X}\right):_{\omega_{A}} I^{r}
$$

as submodules of $\omega_{A}$ for all $n \geq 0$. For details, see 24] Proposition 2.3].

Now we note that because $J$ is generated by a regular sequence,

$$
J^{n} \omega_{A}:_{A} \omega_{A}=J^{n}
$$

for all $n \geq 0$. This can be proved by induction on $n$. When $n=1$, this follows from the fact that $\omega_{A / J}=\omega_{A} / J \omega_{A}$ is a faithful $A / J$-module. Suppose then that $n>1$. Let $x \in J^{n} \omega_{A}: \omega_{A}$. By the induction hypothesis, we know that $x \in J^{n-1}$. There thus exists a form $F \in A\left[t_{1}, \ldots, t_{r}\right]$ of degree $n-1$ such that $x=F\left(a_{1}, \ldots, a_{r}\right)$. As $F\left(a_{1}, \ldots, a_{r}\right) \omega_{A} \in J^{n} \omega_{A}$ and $\left(a_{1}, \ldots, a_{r}\right)$ is an $\omega_{A}$-regular sequence, every coefficient of $F$ must lie in $J \omega_{A}: \omega_{A}=J$. Hence $x \in J^{n}$.

Finally, to see that

$$
\Omega_{h}:_{A} \omega_{A}=J^{r+1}:_{A} I^{r},
$$

we simply compute

$$
\Omega_{h}:_{A} \omega_{A}=\left(J^{r+1} \omega_{A}: \omega_{A} I^{r}\right):_{A} \omega_{A}=\left(J^{r+1} \omega_{A}:_{A} \omega_{A}\right):_{A} I^{r}=J^{r+1}: I^{r} .
$$

Here, the first equality follows from (11) above, and the last equality follows from (12).

Proof that Theorem 5.1.4 implies Corollary 5.1.1. Say that $y \in \operatorname{core}(I)$. Then

$$
y \omega_{A} \subset \operatorname{core}\left(I \omega_{A}\right)=\Omega_{h}
$$

by Theorem [5.1.4] so $y \in \Omega_{h}:_{A} \omega_{A}=J^{r+1}:_{A} I^{r}$. Conversely, say that

$$
y \in \Omega_{h}:_{A} \omega_{A}=J^{r+1}:_{A} I^{r} .
$$

Then $y \in J^{r+1}:_{A} J^{r}=J$ for every reduction $J$ of $I$, so $y \in \operatorname{core}(I)$. The proof of 5.1.1 is complete. 
Proof of Theorem 5.1.4 Assume that $I$ is equimultiple. As usual, write $\Omega_{h}$ for $\Gamma\left(Y, I^{h} \omega_{Y}\right)$, considered as a submodule of $\omega_{A}$. To see that $\Omega_{h} \subset \operatorname{core}\left(I \omega_{A}\right)$, recall that $\Omega_{h}=J^{r+1} \omega_{A}: \omega_{A} I^{r}$ (as in the proof of Lemma 5.1.6). Thus $\Omega_{h} \subset J^{r+1} \omega_{A}: \omega_{A}$ $J^{r} \subset J \omega_{A}$, since $J$ is generated by a regular sequence.

It remains to show that core $\left(I \omega_{A}\right) \subset \Omega_{h}$. Fix elements $x_{1}, \ldots, x_{d-h} \in A$ such that $\left(x_{1}+I, \ldots, x_{d-h}+I\right)$ is a system of parameters on $A / I$ and a regular sequence of degree zero elements on $G$, the associated graded ring of $A$ with respect to $I$ (see e.g. [19] Proposition 10.24 and its proof]). Then also $\left(x_{1}^{t}+I, \ldots, x_{d-h}^{t}+I\right)$ is a regular sequence on $G$. Set $\bar{A}=A /\left(x_{1}^{t}, \ldots, x_{d-h}^{t}\right)$ and let $\bar{I}$ denote the image of $I$ in $A$. Then $\bar{I}$ is an $\overline{\mathfrak{m}}$-primary ideal of $\bar{A}$. Moreover, the corresponding associated graded ring

$$
\bar{G}=\operatorname{gr}_{\bar{I}} \bar{A}:=\frac{\bar{A}}{\bar{I}} \oplus \frac{\bar{I}}{\bar{I}^{2}} \oplus \ldots
$$

is easily seen to be isomorphic to

$$
G /\left(x_{1}^{t}+I, \ldots, x_{d-h}^{t}+I\right),
$$

and hence Cohen-Macaulay. (For example, one can use the fact that $\left(x_{1}^{t}, \ldots, x_{d-h}^{t}\right) \cap$ $I^{n}=\left(x_{1}^{t}, \ldots, x_{d-h}^{t}\right) I^{n}$ for all $n \geq 0$; see e. g. 19. Theorem 13.10 and Theorem 13.7]).

Furthermore, because $\bar{G}$ is obtained from $G$ by killing elements of degree zero, the $a$-invariants of $G$ and $\bar{G}$ are equal (see Remark 2.6.4 here, or 45] Remark 5.1.21]). Because the Rees ring $A[I t]$ is Cohen-Macaulay, the $a$-invariants $a(G)$ and $a(\bar{G})$ are negative. So because $\bar{G}$ is Cohen-Macaulay with negative $a$-invariant, the corresponding Rees ring

$$
\bar{R}=\bar{A} \oplus \bar{I} \oplus \bar{I}^{2} \ldots
$$

is Cohen-Macaulay. So Theorem 3.0 .2 can be applied to the $\overline{\mathfrak{m}}$-primary ideal $\bar{I}$ in the Cohen-Macaulay ring $\bar{A}$.

Now observe that every minimal reduction of $\bar{I}$ is of type can be obtained as the image $\bar{J}$ in $\bar{A}$ of some minimal reduction $J$ of $I$. Indeed, let $\left(\overline{a_{1}}, \ldots, \overline{a_{h}}\right)$ be a minimal reduction of $\bar{I}$ with $a_{1}, \ldots, a_{h} \in I$. Then $\bar{I}^{n+1}=\left(\overline{a_{1}}, \ldots, \overline{a_{h}}\right) I^{n}$ for some $n \geq 0$ implies

$$
\begin{aligned}
I^{n+1} & \subset\left(a_{1}, \ldots, a_{h}\right) I^{n}+\left(x_{1}^{t}, \ldots, x_{d-h}^{t}\right) \cap I^{n+1} \\
& =\left(a_{1}, \ldots, a_{h}\right) I^{n}+\left(x_{1}^{t}, \ldots, x_{d-h}^{t}\right) I^{n+1}
\end{aligned}
$$

so that $I^{n+1} \subset\left(a_{1}, \ldots, a_{h}\right) I^{n}$. It thus follows that $\left(a_{1}, \ldots, a_{h}\right)$ is a minimal reduction of $I$.

Finally, let $y \in \operatorname{core}\left(I \omega_{A}\right)$. Since $\omega_{\bar{A}}=\frac{\omega_{A}}{\left(x_{1}^{t}, \ldots, x_{d-h}^{t}\right) \omega_{A}}$, the above computation shows that $\bar{y} \in \operatorname{core}\left(\bar{I} \omega_{\bar{A}}\right)$. Set $\bar{Y}=\operatorname{Proj} \bar{R}$. Then by Theorem 3.0.2 together with Proposition 4.1.4 we have

$$
\operatorname{core}\left(\bar{I} \omega_{\bar{A}}\right) \subset \Gamma\left(\bar{Y}, \omega_{\bar{Y}}(h)\right) .
$$

Now, again using the injection $\Gamma\left(\bar{Y}, \omega_{\bar{Y}}(n)\right) \hookrightarrow \omega_{\bar{A}}$ induced by the trace map, one easily checks that there is an induced isomorphism

$$
\Gamma\left(\bar{Y}, \omega_{\bar{Y}}(n)\right) \cong \frac{\Omega_{n}}{\left(x_{1}^{t}, \ldots, x_{d-h}^{t}\right) \Omega_{n}}
$$


for all $n \in \mathbb{Z}$. Thus

$$
\bar{y}:=y \bmod \left(x_{1}^{t}, \ldots, x_{d-h}^{t}\right) \omega_{A} \in \frac{\Omega_{h}}{\left(x_{1}^{t}, \ldots, x_{d-h}^{t}\right) \Omega_{h}} \subset \frac{\omega_{A}}{\left(x_{1}^{t}, \ldots, x_{d-h}^{t}\right) \omega_{A}},
$$

So

$$
y \in \Omega_{h}+\left(x_{1}^{t}, \ldots, x_{d-h}^{t}\right) \omega_{A} .
$$

Finally, because this works for any positive $t$, we have

$$
y \in \bigcap_{t \geq 1}\left(\Omega_{h}+\left(x_{1}^{t}, \ldots, x_{d-h}^{t}\right) \omega_{A}\right)=\Omega_{h} .
$$

This shows that core $\left(I \omega_{A}\right) \subset \Omega_{h}$, and the proof is complete.

5.2. Core in Dimension one. The main technical theorem easily gives a formula for the core of an m-primary ideal in a local ring of dimension one, without any Cohen-Macaulay hypothesis at all.

Corollary 5.2.1. Let $(A, \mathfrak{m})$ be a one dimensional local ring containing the rational numbers. Then for any $\mathfrak{m}$-primary ideal I, we have

$$
\operatorname{core}\left(I \omega_{A}\right)=\Omega_{1} .
$$

In particular, if $A$ is Cohen-Macaulay, then

$$
\operatorname{core}(I)=J^{r+1}: I^{r},
$$

where $J$ is any reduction of $I$ and $r$ is any positive integer such that $I^{r+1}=J I^{r}$.

Proof. The second statement follows immediately from the first using Lemma 5.1.6. The first statement follows immediately from the the main technical theorem, Theorem 3.0.2 One need only verify that $\Omega_{1} \subset J \omega_{A}$ and $J \omega_{A} \cap \Omega_{0}=J\left(\Omega_{-1} \cap \omega_{A}\right)$, but this is trivial by Lemma 3.1.4 since $J$ is generated by a non-zero-divisor on $\omega_{A}$.

5.3. Core and adjoints. We recall the definition of an adjoint (or multiplier) ideal. Although a definition can be given that does not refer to resolution of singularities (see 35), we prefer the following approach.

Let $X$ be a Gorenstein scheme essentially of finite type over a field of characteristic zero, and let $\mathfrak{a}$ be a coherent sheaf of ideals on $X$. Fix a $\log$ resolution of $\mathfrak{a}$, that is, a proper birational map $Y \stackrel{\pi}{\rightarrow} X$ from a smooth scheme $Y$ such that $\mathfrak{a} \mathcal{O}_{Y}$ is locally principal and the union of the support of the corresponding divisor and the exceptional divisors is a divisor with normal crossing support. Then the multiplier (or adjoint) ideal of $\mathfrak{a}$ is the ideal sheaf of $\mathcal{O}_{X}$

$$
\operatorname{adj}(\mathfrak{a})=\pi_{*}\left(\mathfrak{a} \omega_{Y / X}\right),
$$

where $\omega_{Y / X}=\omega_{Y} \otimes \pi^{*} \omega_{X}$ is the relative canonical sheaf of $\pi$. This is independent of the choice of the log resolution. Note that because $X$ is Gorenstein, $\omega_{X}$ is invertible, so $\omega_{Y / X}$ is invertible as well. See 31] or [9] for the general theory of multiplier ideals from the algebro-geometric point of view, or 35] for a more algebraic point of view.

In 22], Huneke and Swanson studied the core of an integrally closed $\mathfrak{m}$-primary ideal in a two-dimensional regular local ring. In particular, they showed that in this case,

$$
\operatorname{core}(I)=\operatorname{adj}\left(I^{2}\right)=I \operatorname{adj}(I) .
$$

However, such ideals are very special in a sense: the corresponding Rees algebra always has rational singularities (see [33, Proposition 1.2] and [23, Proposition 2.1]). 
In particular, it is Cohen-Macaulay and normal. For this reason the following can be considered a natural generalization of their result to higher dimension.

Corollary 5.3.1. Let $A$ be regular local ring essentially of finite type over a field of characteristic zero. Let $I \subset A$ be equimultiple ideal of positive height $h$ such that the Rees ring $A[I t]$ is normal and Cohen-Macaulay. Then the following conditions are equivalent

1) $A[I t]$ has rational singularities;

2) $\Omega_{n}=\operatorname{adj}\left(I^{n}\right)$ for all $n \geq 0$;

3) $\operatorname{core}(I)=\operatorname{adj}\left(I^{h}\right)$.

If this is the case, then

$$
\operatorname{core}(I)=I \operatorname{adj}\left(I^{h-1}\right) \text { and } \operatorname{adj}\left(I^{h-1}\right)=\operatorname{core}(I): I .
$$

Remark 5.3.2. In fact, as is clear from the proof, it is not necessary that $A$ be regular for this theorem. It is sufficient if $A$ is Gorenstein with rational singularities.

Remark 5.3.3. In dimension two it is not necessary that $A$ is essentially of finite type over a field of characteristic zero. Indeed, resolutions exist in this setting, and the hypotheses on the Rees ring imply that the reduction number is at most one; see Remark 3.0.4 In particular, since the hypotheses on the Rees ring hold automatically for any $\mathfrak{m}$-primary integrally closed ideal in a two regular local dimensional local ring, the Huneke-Swanson theorem is recovered in full generality.

Proof. Set $Y=\operatorname{Proj} A[I t]$. Observe first that $A[I t]$ has rational singularities if and only if $Y$ has rational singularities (see for example, 32, Proposition 1.2] and 23, Proposition 2.1]). Let $f: Z \longrightarrow Y$ be a $\log$ resolution of $I \mathcal{O}_{Y}$. Because $Y$ is CohenMacaulay and normal, it follows that $Y$ has rational singularities if and only if the natural inclusion

$$
f_{*} \omega_{Z} \subset \omega_{Y}
$$

is an isomorphism; 28. On the other hand, because $I \mathcal{O}_{Y}$ is ample for the map $Y \longrightarrow \operatorname{Spec} A$, this equivalent to requiring that the natural map

$$
\Gamma\left(Y, I^{n} \mathcal{O}_{Y} \otimes f_{*} \omega_{Z}\right) \hookrightarrow \Gamma\left(Y, I^{n} \omega_{Y}\right)
$$

be an isomorphism for all $n \gg 0$. Because $I^{n} \mathcal{O}_{Y} \otimes f_{*} \omega_{Z}$ can be identified with $f_{*}\left(I^{n} \omega_{Z}\right)$, this is the same as the the natural inclusion

$$
\operatorname{adj}\left(I^{n}\right)=\Gamma\left(Z, I^{n} \omega_{Z}\right) \hookrightarrow \Gamma\left(Y, I^{n} \omega_{Y}\right)=\Omega_{n}
$$

being an isomorphism for all $n \gg 0$.

Now, by Lipman's Briançon-Skoda Theorem (see also [31)

$$
\operatorname{adj}\left(I^{h}\right)=I \operatorname{adj}\left(I^{h-1}\right)
$$

and by our Briançon-Skoda Theorem (actually Corollary 4.1.2 and the subsequent remark)

$$
\Omega_{h}=I \Omega_{h-1} .
$$

So remembering also that $\Omega_{n+1}: \omega_{A} I=\Omega_{n}$ for all $n \geq 0$, by Lemma 3.1.5 we conclude that (13) is an isomorphism for all $n \gg 0$ if and only if it is an isomorphism for $n=h-1$. This proves the equivalence of statements (1) and (2). The equivalence with (3) is also clear, since core $(I)=\Omega_{h}$ by Theorem 5.1.4

Finally, the formula $\operatorname{adj}\left(I^{h-1}\right)=\operatorname{core}(I): I$ is a consequence of the formula $\Omega_{h-1}=\Omega_{h}:_{A} I$ of Lemma 3.1.5 The corollary is proved. 
Example 5.3.4. Let $I$ be a normal equimultiple monomial ideal of height $h$ in a polynomial ring $S$ over $\mathbb{C}$. Then

$$
\text { core } I=\operatorname{adj}\left(I^{h}\right)=I \operatorname{adj}\left(I^{h-1}\right) .
$$

Indeed, in this case, the Rees ring $S[I t]$ is a normal semi-group algebra, and hence has rational singularities (since it is a direct summand of a polynomial ring [3]). In particular, if $I$ is generated by monomials $x^{A_{i}}=x_{1}^{a_{i 1}} x_{2}^{a_{i 2}} \ldots x_{n}^{a_{i d}}$, then core $(I)$ is generated by monomials $x^{B}=x_{1}^{b_{1}} \ldots x_{d}^{b_{d}}$ where

$$
\left(b_{1}+1, b_{2}+1, \ldots, b_{d}+1\right)
$$

is in the interior of the convex hull of the points $h A_{1}, \ldots, h A_{r}$ in $\mathbb{N}^{d}$; 21].

5.4. Core and coefficient ideals. If $J \subset I$ is a reduction of $I$, Aberbach and Huneke defined the coefficient ideal $\mathfrak{a}(I, J)$ as the largest ideal $\mathfrak{a}$ such that $I \mathfrak{a}=J \mathfrak{a}$; see [2]. The next corollary relates this notion to the core.

Corollary 5.4.1. Let $(A, \mathfrak{m})$ be a Gorenstein local ring containing the set of rational numbers. Let $I \subset A$ be an equimultiple ideal of positive height $h$ such that the Rees ring $A[I t]$ is Cohen-Macaulay. If $J \subset I$ is any minimal reduction, then

$$
\operatorname{core}(I)=I \mathfrak{a}(I, J) \quad \text { and } \quad \mathfrak{a}(I, J)=\operatorname{core}(I): I .
$$

Proof. By Theorem 5.1.4 we know that $\operatorname{core}(I)=\Omega_{h}$. By the Briançon-Skoda Theorem (Lemma 4.1.1),$\Omega_{h}=I \Omega_{h-1}$. On the other hand, according to 24. Theorem 3.4], $\Omega_{h-1}=\mathfrak{a}(I, J)$. Thus the first claim follows. The second one is now a consequence of the formula $\Omega_{h-1}=\Omega_{h}: I$ of Lemma 3.1.5

Remark 5.4.2. In fact, rational singularities of $A[I t]$ can be characterized in terms of the equality $\mathfrak{a}(I, J)=\operatorname{adj}\left(I^{h-1}\right)$ where $J \subset I$ is any minimal reduction; See 24, Corollary 3.5].

\subsection{Further Properties of Core, and Questions.}

Corollary 5.5.1. Let $A$ be a Gorenstein local ring essentially of finite type over a field of characteristic zero. Let $I$ be an equimultiple ideal of positive height $h$ such that the Rees ring $A[I t]$ has rational singularities. Then

$$
\operatorname{core}(I) \subset \operatorname{core}\left(I^{\prime}\right)
$$

for any ideal $I^{\prime}$ of height $h$ containing $I$.

Proof. Because $I \subset I^{\prime}$, we know $\operatorname{adj}\left(I^{h}\right) \subset \operatorname{adj}\left(\left(I^{\prime}\right)^{h}\right)$. By Corollary [5.3.1] we have core $(I)=\operatorname{adj}\left(I^{h}\right) \subset \operatorname{adj}\left(\left(I^{\prime}\right)^{h}\right)$. On the other hand, from the BriançonSkoda theorem $([35]), \operatorname{adj}\left(\left(I^{\prime}\right)^{h}\right)$ is contained in every reduction of $I^{\prime}$. So core $(I) \subset$ $\operatorname{core}\left(I^{\prime}\right)$.

Question 5.5.2. If $I$ is an integrally closed ideal, then is core $(I) \subset \operatorname{core}\left(I^{\prime}\right)$ for all ideals $I^{\prime}$ containing $I$ ? If $I$ is not integrally closed, the answer is no in general. Indeed, whenever $I \subset I^{\prime}$ is an integral extension of ideals, then a minimal reduction of $I$ is a minimal reduction of $I^{\prime}$, but $I^{\prime}$ may admit reductions that are not reductions of $I$. So clearly core $\left(I^{\prime}\right) \subset \operatorname{core}(I)$, but the inclusion can be strict, and usually is, for example, when $I$ is a minimal reduction of $I^{\prime}$. On the other hand, the same reasoning indicates that there is no loss of generality in assuming that also that $I^{\prime}$ is integrally closed in Question 5.5.2 This question was first raised in 22.

The next result has to do with when the core itself is integrally closed. 
Proposition 5.5.3. Let $A$ be a Cohen-Macaulay local domain of dimension d containing the set of rational numbers. Let $I \subset A$ be a normal equimultiple ideal of positive height $h$ such that the Rees ring $A[I t]$ is Cohen-Macaulay. Then core $(I)$ is an integrally closed ideal of $A$.

Proof. Set $Y=\operatorname{Proj} A[I t]$. Consider $\omega_{Y}$ as a sub-sheaf of the constant sheaf $K$ where $K$ is the quotient field of $A$. Since $\omega_{Y}$ is reflexive, we know that

$$
\Omega_{h}=\Gamma\left(Y, I^{h} \omega_{Y}\right)=\bigcap_{\operatorname{codim} \frac{1}{\{x\}}=1} I^{h} \omega_{Y, x}
$$

But then

$$
\operatorname{core}(I)=\Omega_{h}:_{A} \omega_{A}=\bigcap_{\operatorname{codim}} \frac{I_{\{x\}}=1}{I^{h} \omega_{Y, x}:_{A} \omega_{A} .}
$$

Thus core $(I)$ is integrally closed, because it is an intersection of integrally closed ideals of $A$.

Question 5.5.4. Under what conditions is the core of a normal ideal integrally closed? This issue was first raised in [22. See also [7, Examples 3.9 and 3.10].

Finally, we record an observation about the asymptotic behavior of core, as a partial answer to a question raised in 22$]$.

Corollary 5.5.5. Let $(A, \mathfrak{m})$ be a Gorenstein local ring containing the rational numbers. Let $I \subset A$ be an equimultiple ideal of positive height $h$ such that $A[I t]$ is Cohen-Macaulay. Then

$$
\operatorname{core}\left(I^{n}\right)=I^{(n-1) h} \operatorname{core}(I)
$$

for all $n \geq 1$.

Proof. Set $Y=\operatorname{Proj} A\left[I^{n} t\right]$. Since $Y=\operatorname{Proj} R^{(n)} \cong \operatorname{Proj} R$, we observe that $\Gamma\left(Y, I^{n k} \omega_{Y}\right)=\Omega_{k n}$ for all $k \in \mathbb{Z}$. The ideal $I^{n}$ being also equimultiple of height $h$, Theorem 5.1.4 and Corollary 4.1.2 (and the subsequent remark), now give

$$
\operatorname{core}\left(I^{n}\right)=\Gamma\left(Y, I^{n h} \omega_{Y}\right)=\Omega_{n h}=I^{n h-h} \Omega_{h}=I^{(n-1) h} \operatorname{core}(I) .
$$

\section{Non-Vanishing Sections and the Core}

The goal of this section is to reduce Kawamata's Conjecture to a purely algebraic statement relating the core of an $\mathfrak{m}$-primary ideal in a local ring of dimension $d$ to the adjoint ideal (or multiplier ideal) of the $d$-th power of the ideal. Actually, of course, we must work in the graded category. Also, to get at the most general version of Conjecture 1.1.1 we must expand the notions of the core and the adjoint to submodules of the canonical module. The main result of this section is the following theorem.

Theorem 6.0.6. Let $D$ be an ample Cartier divisor on a rationally singular projective variety $X$ of positive dimension, and let

$$
S=\bigoplus_{n \geq 0} H^{0}(X, n D)
$$


be the corresponding section ring. Fix $n \gg 0$, and let $I=S_{\geq n}$ be the ideal generated by all elements of degrees at least $n$ in $S$. Then $H^{0}(X, D) \neq 0$ if

$$
\text { gradedcore }\left(I \omega_{S}\right)=\operatorname{adj}\left(I^{d+1} \omega_{S}\right), \quad \text { where } d+1=\operatorname{dim} S,
$$

as subsets of $\omega_{S}$.

Remark 6.0.7. Here, gradedcore $\left(I \omega_{S}\right)$ denotes the intersection of all submodules of $\omega_{S}$ of the form $J \omega_{S}$, where $J$ is a homogeneous reduction of $I$. Likewise, for any ideal $I$ in a normal domain $S, \operatorname{adj}\left(I \omega_{S}\right)$ denotes the following natural variant of the adjoint ideal. Fix a $\log$ resolution $Y \rightarrow \operatorname{Spec} S$ of $I$. Then $\operatorname{adj}\left(I \omega_{S}\right)$ is the submodule of $\omega_{S}$ given by $\pi_{*}\left(I \omega_{S}\right)$. This definition is independent of the choice of $\log$ resolution. This is like the usual notion of multiplier ideal, but the relative canonical modules has been replaced by the absolute canonical module of $Y$. This has the advantage of being defined even when $S$ is not Gorenstein (or $\mathbb{Q}$-Gorenstein). However, it is a submodule of $\omega_{S}$ rather than an ideal of $S$.

Remark 6.0.8. Formula (14) is a graded version of the formula we proved (under certain conditions on the ring and ideal) in Section 5. As we will see, rings arising from divisors satisfying the hypothesis of Conjecture 1.1.1 satisfy these conditions, so Kawamata's Conjecture is very closely related to our formulas in 5.3.1

Remark 6.0.9. As will be clear from the proof, a version of Theorem 6.0.6 holds if $X$ is not necessarily rationally singular, but is only normal. In this case, $H^{0}(X, D)$ is non-zero if gradedcore $\left(I \omega_{S}\right)=\Omega_{d+1}$, with notation as in Section 3 .

Remark 6.0.10. In fact, the converse of Theorem 6.0.6 is also true: $H^{0}(X, D)$ is non-zero if and only if the formula (14) holds for $n \gg 0$ in the section ring $S$ of $D$. However, the proof of the requires rather different ideas and techniques, so we postpone it to a subsequent paper.

In this section, we first prove Theorem 6.0.6. We then investigate the hypothesis forced upon the section ring $S$ of a pair $(X, D)$ satisfying the hypothesis of Conjecture 1.1.1. Finally, we end with a discussion of core versus graded core in a graded ring.

6.1. A general criterion for non-vanishing. Let $D$ be an ample Cartier divisor on a normal projective variety $X$ of dimension $d \geq 1$. By definition, the section ring of the pair $(X, D)$ is the $\mathbb{N}$-graded ring

$$
S=\bigoplus_{n \in \mathbb{N}} H^{0}\left(X, \mathcal{O}_{X}(n D)\right),
$$

whose multiplication is given by the natural multiplication of sections. The ring $S$ is a normal graded domain, finitely generated over the field $k=H^{0}\left(X, \mathcal{O}_{X}\right)$, which we will assume to be infinite. There is a canonical isomorphism from $X$ to Proj $S$ under which the invertible sheaf $\mathcal{O}_{X}(n D)$ corresponds to the coherent module on Proj $S$ arising from the graded $S$-module $S(n)$, where $S(n)$ denotes the module $S$ with its grading shifted so that $S(n)_{m}=S_{m+n}$. For a general reference on section rings, see [16 Section 4.5].

Proposition 6.1.1. Let $D$ be an ample divisor on a normal projective variety $X$, and let $S$ be the corresponding section ring of the pair $(X, D)$. Fix $n \gg 0$. Then

$$
H^{0}(X, D)=0
$$


if and only if

$$
\left[\omega_{S}\right]_{n(d+1)-1} \subset \text { gradedcore }\left(I \omega_{S}\right),
$$

where $\omega_{S}$ is the (graded) canonical module of the normal ring $S$, and I is the ideal of $S$ generated by elements of degrees at least $n$.

Proposition 6.1.1 follows readily from the following very general criterion for the vanishing of the space of global sections of an ample line bundle.

Lemma 6.1.2. Let $D$ be an ample Cartier divisor on a normal projective variety $X$ of dimension $d \geq 1$. Fix any integer $i$. Then $H^{0}(X, i D)=0$ if and only if for some (equivalently, every) $n \gg 0$, and any set $x_{0}, \ldots, x_{d}$ of $d+1$ global generators for $\mathcal{O}_{X}(n D)$, the natural inclusion

$$
\sum_{i=0}^{d} x_{i} H^{0}\left(X, K_{X}+(n d-i) D\right) \subset H^{0}\left(X, K_{X}+[n(d+1)-i] D\right)
$$

is an equality. ${ }^{5}$

Proof of Lemma 6.1.2 Fix any set of $d+1$ global generators for $\mathcal{O}_{X}(n D)$. Such a set always exists (assuming $X$ to be defined over an infinite field), because we can take generic linear combinations of any set of global generators for $\mathcal{O}_{X}(n D)$.

Consider the Koszul complex determined by the $x_{i}$ 's:

$$
0 \longrightarrow \mathcal{O}_{X}(-(d+1) n D) \longrightarrow \ldots \longrightarrow \bigoplus_{i=0}^{d} \mathcal{O}_{X}(-n D) \stackrel{\left(\begin{array}{c}
x_{0} \\
\vdots \\
x_{d}
\end{array}\right)}{\longrightarrow} \mathcal{O}_{X} \longrightarrow 0
$$

Because the $x_{i}$ 's generate $\mathcal{O}_{X}(n D)$, this complex is exact. Tensoring with the invertible sheaf $\mathcal{O}_{X}\left(K_{X}+[(d+1) n-i] D\right)$, we get an exact complex

$0 \longrightarrow \mathcal{O}_{X}\left(K_{X}-i D\right) \longrightarrow \ldots \longrightarrow \bigoplus_{i=0}^{d} \mathcal{O}_{X}\left(K_{X}+(d n-i) D\right) \stackrel{\left(\begin{array}{c}x_{0} \\ \vdots \\ x_{d}\end{array}\right)}{\longrightarrow} \mathcal{O}_{X}\left(K_{X}+[(d+1) n-i] D\right) \longrightarrow 0$

Because $H^{i}\left(X, \mathcal{O}_{X}\left(K_{X}+m D\right)\right)=0$ for all $m \geq n-i$ and all $i>0$, a standard argument ${ }^{6}$ shows that the map of global sections

$$
\bigoplus_{i+0}^{d} H^{0}\left(X, K_{X}+(d n-i) D\right) \stackrel{\left(\begin{array}{c}
x_{0} \\
\vdots \\
x_{d}
\end{array}\right)}{\longrightarrow} H^{0}\left(X, K_{X}+[(d+1) n-i] D\right)
$$

\footnotetext{
${ }^{5}$ The precise condition on $n$ in Propositions 6.1.1 and 6.1.2 is that $n$ should be large enough that $\mathcal{O}_{X}(n D)$ is globally generated and $H^{i}\left(X, K_{X}+m D\right)$ vanishes for all $i>0$ and all $m \geq n-i$.

${ }^{6}$ The standard argument is this: break the complex into several short exact complexes of sheaves. Then look at the corresponding long exact complexes of cohomology, beginning with the 0 -th cohomology of the short exact sequence arising from the right-most part of the complex. Working backwards, the relevant cohomology is the $i$-th cohomology of the $i$-th short exact sequence from the right. A similar argument is written down in full in the proof of Proposition 4.1 .4
} 
is surjective if and only if $H^{d}\left(X, K_{X}-i D\right)=0$.

By Serre duality (which holds at the "top spot" even if $X$ is not Cohen-Macaulay), the claim that $H^{0}(X, i D)$ is zero is identical to the claim that $H^{d}\left(X, K_{X}-i D\right)$ is zero. So $H^{0}(X, D)$ vanishes if and only if

$$
H^{0}\left(X, K_{X}+[n(d+1)-i] D\right) \subset\left(x_{0}, \ldots, x_{d}\right) H^{0}\left(X, K_{X}+[n d-i] D\right) .
$$

The proof of Lemma 6.1.2 is complete.

Proof of Proposition 6.1.1 Interpret global sections of $\mathcal{O}_{X}(n D)$ as degree $n$ elements of $S$. Because a set of global sections $\left\{x_{i}\right\}$ generates $\mathcal{O}_{X}(n D)$ if and only if their common zero set on $X=\operatorname{Proj} S$ is empty, such a set is a generating set for $\mathcal{O}_{X}(n D)$ if and only if the elements $\left\{x_{i}\right\}$ in $S$ generate an $\mathfrak{m}$-primary ideal of $S$. In particular, a set of $d+1$ global sections of $\mathcal{O}_{X}(n D)$ is a generating set if and only if its elements form a homogeneous system of parameters for the ring $S$.

Now fix $n \gg 0$. If $H^{0}(X, D)$ is zero, then for each set $\left\{x_{0}, x_{1}, \ldots, x_{d}\right\}$ of global generators of $\mathcal{O}_{X}(n D)$, Lemma 6.1.2 ensures that

$$
\sum_{i=0}^{d} x_{i} H^{0}\left(X, K_{X}+(n d-1) D\right)=H^{0}\left(X, K_{X}+(n(d+1)-1) D\right) .
$$

Interpreted in terms of the section ring $S$, this says

$$
\left(x_{0}, \ldots, x_{d}\right)\left[\omega_{S}\right]_{n d-1}=\left[\omega_{S}\right]_{n(d+1)-1} .
$$

In particular,

$$
\left[\omega_{S}\right]_{n(d+1)-1} \subset\left(x_{0}, \ldots, x_{d}\right) \omega_{S}
$$

for every system of parameters for $S$ consisting of elements of degree $n$. In other words,

$$
\left[\omega_{S}\right]_{n(d+1)-1} \subset \bigcap_{J \text { s.o.p degree } n .} J \omega_{S},
$$

where $J$ ranges over all homogeneous systems of parameters for $S$ consisting of elements of degree $n$. By Proposition 2.1.3 a system of parameters of degree $n$ is precisely the same as a minimal homogeneous reduction for the ideal $I=S_{\geq n}$, the ideal generated by all elements of degrees at least $n$, so this means that

$$
\left[\omega_{S}\right]_{n(d+1)-1} \subset \text { gradedcore }\left(I \omega_{S}\right) .
$$

The proof of the converse simply reverses this argument.

Remark 6.1.3. Although Proposition 6.1.1 follows quite trivially from Lemma 6.1.2. the passage to this more algebraic lemma seems powerful. The point is that there is hope for showing that the intersection over all submodules of the form $\left(x_{0}, \ldots, x_{d}\right) \omega_{S}$ is quite small, so small in fact, that it can not contain any element of degree $n(d+1)-1$ (although each individual module $\left(x_{0}, \ldots, x_{d}\right) \omega_{S}$ certainly contains many such elements!). This would settle Kawamata's Conjecture if it could be accomplished. 
6.2. An Adjoint Computation. The next proposition is a very general computation of adjoint modules for certain types of ideals in a section ring.

Proposition 6.2.1. Let $S$ be a section ring of a pair $(X, D)$ consisting of an ample Cartier divisor on a normal projective variety. Fix $n \gg 0$ and let $I=S_{\geq n}$ be the ideal of $S$ generated by all elements of degrees at least $n$. Then

$$
\Gamma\left(Y, I \omega_{Y}\right)=\left[\omega_{S}\right]_{\geq n+1},
$$

as submodules of $\omega_{S}$, where $Y$ is the blowup of the scheme $\operatorname{Spec} S$ along the ideal $I$. (The precise condition on $n$ is that $n$ should be large enough that $\left(S_{\geq n}\right)^{t}=S_{\geq t n}$ for all $t \geq 0$.)

The proof of Proposition 6.2.1 makes use of the "natural" construction from 16, Section 8.7.3].

6.2.1. The "natural" construction. Let $S^{\natural}$ be the graded ring

$$
S^{\natural}=S \oplus S_{\geq 1} \oplus S_{\geq 2} \oplus S_{\geq 3} \ldots,
$$

where $S_{\geq n}$ indicates the ideal of $S$ generated by elements of degree at least $n$. The $\operatorname{ring} S^{\natural}$ is finitely generated over its degree zero part $S$, so for large $n$, we have $\left(S_{\geq n}\right)^{k}=S_{\geq n k}$ for all $k \geq 0$. Since the projective scheme of a graded ring is unchanged under passing to any Veronese sub-ring, we have

$$
\operatorname{Proj} S^{\natural} \cong \operatorname{Proj} S[I t]
$$

where $S[I t]$ is the Rees ring of $S$ with respect to the ideal $I=S_{\geq n}$.

There are two natural geometric interpretations of the scheme Proj $S^{\natural}$. First, the above isomorphism shows that Proj $S^{\natural}$ can be considered as the blowup of the ideal $I$ in the affine scheme Spec $S$. On the other hand, there is a natural isomorphism (see [16, 8.7.3])

$$
\operatorname{Spec}_{X}\left(\mathcal{O}_{X} \oplus \mathcal{O}_{X}(D) \oplus \mathcal{O}_{X}(2 D) \oplus \ldots\right) \stackrel{\cong}{\longrightarrow} \operatorname{Proj}\left(S \oplus S_{\geq 1} \oplus S_{\geq 2} \oplus \ldots\right) .
$$

This allows to interpret Proj $S^{\natural}$ also as the total space of the "tautological" line bundle $\mathcal{O}_{X}(-D)$ on $X$ (or, in some writers' terminology, as the scheme $\mathbb{V}\left(\mathcal{O}_{X}(D)\right)$ ). Correspondingly, there are two natural projections,

$$
\operatorname{Proj} S^{\natural} \stackrel{\pi}{\longrightarrow} \operatorname{Spec} S \quad \operatorname{Proj} S^{\natural} \stackrel{\eta}{\longrightarrow} \operatorname{Proj} S=X .
$$

The first is the blowing up morphism, while the second is the structure map of the line bundle $\mathcal{O}_{X}(-D)$.

Remark 6.2.2. These interpretations of Proj $S^{\natural}$ generalize the following situation. Let $Y$ denote the incidence correspondence

$$
Y=\{(p, \ell) \mid p \in \ell\} \subset \mathbb{C}^{n} \times \mathbb{P}^{n-1},
$$

where $\ell$ is a line through the origin in $\mathbb{C}^{n}$ and $p$ is a point on it. By projecting $Y$ to either $\mathbb{C}^{n}$ or $\mathbb{P}^{n-1}$, respectively, we arrive at either the blowup of the origin in $\mathbb{C}^{n}$ or the structure map of the tautological line bundle on $\mathbb{C}^{n}$. Note that here $Y=\operatorname{Proj} S^{\natural}$ where $S$ is the polynomial ring in $n$ variables. 
Proof of Proposition [6.2.1. Using the interpretation of $Y$ as the total space of the tautological bundle, one easily computes $\omega_{Y}$. Indeed, because $Y \stackrel{\eta}{\rightarrow} X$ is smooth of relative dimension one with $\Omega_{Y / X}=\eta^{*} \mathcal{O}_{X}(D)$, we have

$$
\omega_{Y}=\eta^{*} \omega_{X} \otimes \Omega_{Y / X}=\eta^{*} \omega_{X} \otimes \eta^{*} \mathcal{O}_{X}(D)=\eta^{*}\left(\omega_{X}(D)\right) .
$$

Also, thinking of $S[I t]$ as the $n$-th Veronese sub-ring of the algebra $S^{\natural}$, we see that $I^{t} \mathcal{O}_{Y}=\mathcal{O}_{Y}(n t)=\eta^{*} \mathcal{O}_{X}(n t D)$, where $\mathcal{O}_{Y}(n t)$ is the coherent sheaf on $Y=\operatorname{Proj} S^{\natural}$ corresponding to the graded module $S^{\natural}(n t)$.

Now, noting that the map $Y \stackrel{\eta}{\rightarrow} X$ affine and that $\eta_{*} \mathcal{O}_{Y}=\bigoplus_{i \in \mathbb{N}} \mathcal{O}_{X}(i D)$, we compute

$$
\begin{aligned}
\Gamma\left(Y, I^{t} \omega_{Y}\right) & =\Gamma\left(Y, \eta^{*} \mathcal{O}_{X}(t n D) \otimes \eta^{*}\left(\omega_{X} \otimes \mathcal{O}_{X}(D)\right)\right) \\
& =\Gamma\left(Y, \eta^{*}\left(\omega_{X} \otimes \mathcal{O}_{X}([t n+1] D)\right)\right) \\
& =\Gamma\left(X,\left(\eta_{*} \mathcal{O}_{Y} \otimes\left(\omega_{X} \otimes \mathcal{O}_{X}([t n+1] D)\right)\right)\right. \\
& =\Gamma\left(X,\left(\bigoplus_{i \in \mathbb{N}} \mathcal{O}_{X}(i D)\right) \otimes\left(\omega_{X} \otimes \mathcal{O}_{X}([t n+1] D)\right)\right) \\
& =\bigoplus_{i \in \mathbb{N}} \Gamma\left(X, \omega_{X}([t n+1+i] D)\right. \\
& =\left[\omega_{S}\right]_{\geq t n+1} .
\end{aligned}
$$

This completes the proof of Proposition 6.2.1

Proof of Theorem 6.0.6. Suppose that $H^{0}(X, D)=0$. Then by Proposition 6.1.1] we have that

$$
\left[\omega_{S}\right]_{n(d+1)-1} \subset \text { gradedcore }\left(I \omega_{S}\right) .
$$

If the equality (14) of Theorem 6.0.6 holds, then in fact

$$
\left[\omega_{S}\right]_{n(d+1)-1} \subset \operatorname{adj}\left(I^{d+1} \omega_{S}\right) .
$$

Note that the assumptions of Kawamata's Conjecture force the variety $X$ to have rational singularities. Therefore, the scheme $Y=\operatorname{Proj} S[I t]$ also has rational singularities, because it is the total space of a line bundle over $X$. Furthermore, the ideal $I$ (and its powers) pull back to an invertible sheaf on $Y$ under the birational map $Y \longrightarrow$ Spec $S$ whose support is an irreducible closed subvariety (isomorphic to $X)$. It is easy to check that in this situation, the adjoint of $I^{d+1}$ can be computed from the resolution $Y \longrightarrow$ Spec $S$. If particular, if $H^{0}(X, D)=0$, then

$$
\left[\omega_{S}\right]_{n(d+1)-1} \subset \Gamma\left(I^{d+1} \omega_{Y}\right) .
$$

Finally, since $I^{d+1}=S_{\geq n(d+1)}$, we apply Proposition 6.2 .1 to conclude that

$$
\left[\omega_{S}\right]_{n(d+1)-1} \subset\left[\omega_{S}\right]_{\geq n(d+1)+1} .
$$

This is an obvious contradiction, since $\omega_{S}$ is non-zero in all sufficiently large degrees. Thus $H^{0}(X, D)$ can not vanish and the proof is complete. 
6.3. The section ring. In order to use Theorem 6.0.6 to prove Kawamata's Conjecture, we need to better understand the special conditions imposed on $S$ by the hypothesis of Conjecture 1.1.1 Remarkably, it turns out that just the right condition to deduce a local form of formula (14) is satisfied.

Proposition 6.3.1. Let $S$ be the section ring of a normal projective variety $X$ with respect to an ample Cartier divisor $D$, and let $I$ be the ideal of $S$ generated by elements of degree $n \gg 0$. Assume that there exists an effective $\mathbb{Q}$-divisor $B$ such that the pair $(X, B)$ is Kawamata log terminal and the $\mathbb{Q}$-divisor $D-\left(K_{X}+B\right)$ is big and nef. Then the irrelevant ideal

$$
(S[I t])^{+}:=I \oplus I^{2} \oplus I^{3} \oplus \ldots
$$

of the Rees ring $S[I t]$ is a Cohen-Macaulay $S[I t]$-module.

The proof makes use of the following two lemmas.

Lemma 6.3.2. Let $S$ be a section ring as in Proposition 6.3.1. Then the local cohomology modules of $S$ with support in the unique homogeneous maximal ideal $\mathfrak{m}$ satisfy:

(1) For $i<\operatorname{dim} S$, the graded $S$-modules $H_{\mathfrak{m}}^{i}(S)$ vanish in all degrees $n \neq 0$.

(2) $H_{\mathfrak{m}}^{\operatorname{dim} S}(S)$ vanishes in positive degrees.

Lemma 6.3.3. Let $(A, \mathfrak{m})$ be an arbitrary local ring of dimension $d$ and let $\left\{I_{n}\right\}$ be a Noetherian filtration of A consisting of ideals of positive height. Let

$$
R:=A \oplus I_{1} \oplus I_{2} \oplus \ldots
$$

and

$$
G:=A / I_{1} \oplus I_{1} / I_{2} \oplus I_{2} / I_{3} \oplus \ldots
$$

denote, respectively, the Rees ring and the associated graded ring of $A$ with respect to this filtration, whose unique homogeneous maximal ideals will be denoted $\mathfrak{M}_{R}$ and $\mathfrak{M}_{G}$, respectively. Then the irrelevant ideal of $R$ is a Cohen-Macaulay $R$-module if and only if the following two conditions on $G$ are satisfied:

(1) For $i<d,\left[H_{\mathfrak{M}_{G}}^{i}(G)\right]_{n}=0$ for $n \neq 0$.

(2) $H_{\mathfrak{M}_{G}}^{d}(G)$ vanishes in positive degrees.

Proof of Proposition 6.3.1. Fix $n \gg 0$. Setting $I=S_{\geq n}$ to be the ideal in $S$ generated by all elements of degrees at least $n$, we have $I^{k}=S_{\geq n k}$. So for this $n$, the Rees ring $S[I t]$ is the $n$-th Veronese subring of the Rees ring $S^{\natural}$ formed from the filtration $I_{n}=S_{\geq n}$. The irrelevant ideal of $S[I t]$ is therefore the $n$-th Veronese submodule of the irrelevant ideal of $S^{\natural}$. Thus in order to show that the irrelevant ideal of $S[I t]$ is a Cohen-Macaulay $S[I t]$-module, it is sufficient to prove that the irrelevant ideal of $S^{\natural}$ is Cohen-Macaulay (since the appropriate local cohomology modules for the irrelevant ideal of $S[I t]$ are Veronese submodules of the corresponding local cohomology modules for the irrelevant ideal of $S^{\natural}$ ).

To show that the irrelevant ideal of $S^{\natural}$ is Cohen-Macaulay, note that because the irrelevant ideal is graded, it is enough to check Cohen-Macaulayness after localizing at the unique homogeneous maximal ideal of $S[I t]$. So we may replace $S$ by its localization $A$ at its unique homogeneous ideal, and replace the filtration by its image $\left\{I_{n}\right\}$ in $A$. Note that the associated graded ring of $A$ with respect to this filtration $\left\{I_{n}\right\}$ is canonically isomorphic to $S$. Thus, the Proposition follows immediately from combining the two lemmas. 
Proof of Lemma 6.3.2. First note that because $(X, B)$ is Kawamata log terminal, the variety $X$ has rational singularities (see e.g. [27] Th 1.3.6]). In particular, $X$ is Cohen-Macaulay and Serre duality holds for $X$.

To check statements (1) and (2), we make use of the identifications

$$
\left[H_{\mathfrak{m}}^{i}(S)\right]_{n} \cong H^{i-1}\left(X, \mathcal{O}_{X}(n D)\right)
$$

for each $i \geq 2$ and all $n \in \mathbb{Z}$ (see 2.5.1). Now, for $2 \leq i<\operatorname{dim} S$, the vanishing of $H_{\mathfrak{m}}^{i}(S)$ in negative degree follows from the (dual form of the) Kodaira vanishing theorem applied to the ample divisor $D$. For all $i \geq 2$, the vanishing of $H_{\mathfrak{m}}^{i}(S)$ in positive degree follows from the Kawamata-Viehweg vanishing theorem.

Since $S$ is normal, the local cohomology modules $H_{\mathfrak{m}}^{1}(S)$ and $H_{\mathfrak{m}}^{0}(S)$ are zero in any case, so the Proposition is proved.

Proof of Lemma 6.3.3 Let $R^{+}$denote the irrelevant ideal of $R$. We make use of the following two exact sequences

$$
0 \longrightarrow R^{+} \longrightarrow R \longrightarrow A \longrightarrow 0
$$

and

$$
0 \longrightarrow R^{+}(1) \longrightarrow R \longrightarrow G \longrightarrow 0 .
$$

Because the $R$-module $A$ is concentrated in degree zero, the first sequence gives

$$
\left[H_{\mathfrak{M}_{R}}^{i}\left(R^{+}\right)\right]_{n} \cong\left[H_{\mathfrak{M}_{R}}^{i}(R)\right]_{n}
$$

for all $n \neq 0$ and all $i$. Then looking at the long exact sequence arising from the second sequence, we find a long exact sequence

$$
\ldots \longrightarrow\left[H_{\mathfrak{M}_{R}}^{i-1}(G)\right]_{n} \longrightarrow\left[H_{\mathfrak{M}_{R}}^{i}\left(R^{+}\right)\right]_{n+1} \longrightarrow\left[H_{\mathfrak{M}_{R}}^{i}(R)\right]_{n} \longrightarrow\left[H_{\mathfrak{M}_{R}}^{i}(G)\right]_{n} \longrightarrow \ldots
$$

in each degree $n$.

Assume that $R^{+}$is Cohen-Macaulay. Then for $i<d+1=\operatorname{dim} R^{+}$, we have $\left[H_{\mathfrak{M}_{R}}^{i}\left(R^{+}\right)\right]_{n}=0$ for all $n$. This implies that $\left[H_{\mathfrak{M}_{R}}^{i}(R)\right]_{n}=0$ for all $n \neq 0$. So the long exact sequence above tells us that $H_{\mathfrak{M}_{R}}^{i}(G)$ vanishes in every non-zero degree, for all $i<d$.

For $i=d$, if $n>0$, the long exact sequence above becomes

$$
\longrightarrow\left[H_{\mathfrak{M}_{R}}^{d}(R)\right]_{n} \longrightarrow\left[H_{\mathfrak{M}_{G}}^{d}(G)\right]_{n} \longrightarrow\left[H_{\mathfrak{M}_{R}}^{d+1}\left(R^{+}\right)\right]_{n+1} \longrightarrow 0 .
$$

Because $\left.H_{\mathfrak{M}_{R}}^{d+1}\left(R^{+}\right)\right]_{n+1} \cong\left[H_{\mathfrak{M}_{R}}^{d+1}(R)\right]_{n+1}$, and the $a$-invariant of the Rees ring $R$ is -1 , we see $\left[H_{\mathfrak{M}_{R}}^{d+1}\left(R^{+}\right)\right]_{n+1}=0$ for $n \geq 0$. So both modules

$$
\left[H_{\mathfrak{M}_{R}}^{d}(R)\right]_{n} \text { and }\left[H_{\mathfrak{M}_{R}}^{d+1}\left(R^{+}\right)\right]_{n+1}
$$

are zero for $n>0$, and so $H_{\mathfrak{M}_{G}}^{d}(G)$ vanishes in positive degree. The converse argument just reverses this. The lemma is proved. 
6.4. Core and Graded Core in Graded Rings. We have seen that Kawamata's Conjecture follows from the following conjecture.

Conjecture 6.4.1. Let $S$ be the section ring of a pair $(X, D)$ satisfying the hypothesis of Conjecture 1.1.1. Then

$$
\text { gradedcore }\left(I \omega_{S}\right)=\operatorname{adj}\left(I^{d} \omega_{S}\right)
$$

where $d$ is the dimension of $S$, and $I=S_{\geq n}$ is the $\mathfrak{m}$-primary ideal generated by elements of degrees at least $n$, for some $n \gg 0$.

On the other hand, for a local ring $(A, \mathfrak{m})$ and an ideal $I$ satisfying conditions satisfied by $S_{\geq n}$ in such a section ring, we have proved (see the remark following Theorem 5.1.4 and Proposition 6.3.11 that

$$
\operatorname{core}\left(I \omega_{A}\right)=\operatorname{adj}\left(I^{d} \omega_{A}\right) .
$$

In particular, core $\left(I \omega_{S}\right)=\operatorname{adj}\left(I^{d} \omega_{S}\right)$, for the section ring $S$ of a pair $(X, D)$ satisfying the hypothesis of Kawamata's Conjecture. It is easy to believe that perhaps the core and graded core of $I=S_{\geq n}$ are equal for large $n$, and hence that we have proved Kawamata's Conjecture. However, the problem is appears to be quite subtle. In fact, we have the following corollary of the Main Technical Theorem.

Corollary 6.4.1. Let $S$ be a section ring of a normal Cohen-Macaulay variety of characteristic zero with respect to any ample divisor. Let $I=S_{\geq n}$ be the ideal generated by the homogeneous elements of $S$ of degrees at least $n$, for $n \gg 0$. Then

$$
\operatorname{core}\left(I \omega_{S}\right)=\left[\omega_{S}\right]_{\geq n d+1},
$$

where $d=\operatorname{dim} S$. In particular, if the variety is rationally singular, then

$$
\operatorname{core}\left(I \omega_{S}\right)=\operatorname{adj}\left(I^{d} \omega_{S}\right) .
$$

Furthermore, if $S$ is Cohen-Macaulay,

$$
\operatorname{core}(I)=S_{\geq n d+a+1}
$$

where $a$ is the a-invariant of $S$.

Remark 6.4.2. Of course, since there are ample line bundles on smooth varieties with no sections, Proposition 6.1.1 makes clear that formula (15) can not hold in general. Indeed, given any ample line bundle with no non-zero global sections, one can generate examples of ideals (namely $S_{\geq n}$ for $n \gg 0$ ) in a graded ring (the corresponding section ring) which have many homogeneous reductions, but for which the core is not equal to the graded core.

Proof. The statement may be checked locally at the unique homogeneous maximal ideal of $S$, so we can replace $I$ by its expansion to $A=S_{\mathfrak{m}}$, and our previous results in the local case apply to $S$. As always, we let $Y$ denote the blowup of Spec $S$ along $I$, and set $\Omega_{t}=H^{0}\left(Y, I^{t} \omega_{Y}\right)$. From Proposition 6.2.1 we have

$$
\Omega_{t}=\left[\omega_{S}\right]_{\geq n t+1}
$$

for all $t$. Since $S$ is Cohen-Macaulay on the punctured spectrum, by taking $n \gg 0$, one sees that the irrelevant ideal of $S[I t]$ is Cohen-Macaulay. Thus

$$
J \omega_{S} \cap \Omega_{d-1}=J \Omega_{d-2}
$$


for all reductions $J$ of $I$, by Proposition 4.2.1 As pointed out in Remark 3.0.3 following the Main Technical Theorem, this ensures that

$$
\text { core } I \omega_{S} \subset \Omega_{d}=\left[\omega_{S}\right]_{\geq n d+1} \text {. }
$$

This holds without requiring that $S$ be Cohen-Macaulay, as does the statement core $(I) \subset S_{\geq n d+a+1}$. Indeed, if $z \in \operatorname{core}(I)$ has degree less than $n d+a+1$, then by taking any non-zero element $w$ of $\left[\omega_{S}\right]_{-a}$, we would have an element $y w \in \operatorname{core}\left(I \omega_{S}\right)$ of degree less than $n d+1$, a contradiction.

For the reverse inclusion, we need also that $\Omega_{d} \subset J \omega_{S}$. This follows from the Briançon-Skoda Theorem 4.1.1 because $S$ is Cohen-Macaulay on the punctured spectrum and $n$ is sufficiently large. Indeed, we need only that $H^{i}\left(Y, I^{d-1-i} \omega_{Y}\right)$ is zero for all $i \geq 1$, where as usual $Y=\operatorname{Proj} S[I t]$. For $i<d-1$, this is essentially Serre vanishing (as $n$ is large). For $i=d-1$, the required vanishing holds by Lemma 6.4.3 below. For $i \geq d$, all the cohomology vanishes since $Y$ has a cover by $d$ open affine sets.

The corresponding statement for ideals follows as in the proof that Theorem 5.1 .4 implies 5.1 .1

Lemma 6.4.3. If $I$ is a normal ideal in a normal local ring $A$ of dimension $d$ at least two, then $H^{d-1}\left(Y, \omega_{Y}\right)=0$, where $Y=\operatorname{Proj} A[I t]$.

Proof. Let $Z \stackrel{\nu}{\rightarrow} Y$ be a resolution of singularities of $Y$. The composition $Z \rightarrow Y \rightarrow$ $\operatorname{Spec} A$ is a resolution of singularities of $\operatorname{Spec} A$. We have a short exact sequence

$$
0 \rightarrow \nu_{*} \omega_{Z} \rightarrow \omega_{Y} \rightarrow Q \rightarrow 0
$$

where $Q$ is supported on some set of codimension at least two. This gives rise to a long exact sequence, which-because $\operatorname{dim} Q$ is at most $d-2$ - gives a surjection

$$
H^{d-1}\left(Y, \nu_{*} \omega_{Z}\right) \rightarrow H^{d-1}\left(Y, \omega_{Y}\right) .
$$

On the other hand, by the Grauert-Riemenschneider vanishing theorem $R^{p} \nu_{*} \omega_{Z}=0$ for $p>0$, so the appropriate spectral sequence degenerates to give an isomorphism $H^{i}\left(Y, \nu_{*} \omega_{Z}\right) \cong H^{i}\left(Z, \omega_{Z}\right)$. But again by the Grauert-Riemenschneider vanishing theorem, this time applied to the resolution $Z \rightarrow \operatorname{Spec} A$, we have $H^{i}\left(Z, \omega_{Z}\right)$ vanishes as well for all $i>0$. So $H^{d-1}\left(Y, \nu_{*} \omega_{Z}\right)$ must be zero, and therefore, so is its surjective image $H^{d-1}\left(Y, \omega_{Y}\right)$.

Since every normal standard graded domain is a section ring, we have the following corollary.

Corollary 6.4.4. Let $S$ be normal Cohen-Macaulay $\mathbb{N}$-graded domain finitely generated by its degree one elements over a field of characteristic zero. Let $\mathfrak{m}$ denote its unique homogeneous maximal ideal. Then for all $n>0$,

$$
\operatorname{core}\left(\mathfrak{m}^{n}\right)=\text { gradedcore }\left(\mathfrak{m}^{n}\right)=\mathfrak{m}^{n d+a+1},
$$

where $d=\operatorname{dim} S$ and $a$ is the a-invariant of $S$.

Proof. The formula for core follows from the above corollary since $S_{\geq n}=\mathfrak{m}^{n}$. It remains only verify that the core is the graded core in this situation. Set $I=\mathfrak{m}^{n}$. Looking at the proof of the Key Lemma, we see that gradedcore $\left(I \omega_{S}\right) \subset \Omega_{d}=$ $\left[\omega_{S}\right]_{\geq d n+1}$ if the corresponding intersection $\cap_{x_{1}^{*}, \ldots, x_{d}^{*}}\left(x_{1}^{*}, \ldots, x_{d}^{*}\right) \omega_{G}$ is zero in degree $d$. But because $I$ is generated by elements all of the same degree, this follows from Theorem 3.4.1 
Remark 6.4.5. In Corollary 6.4.4 one can weaken the assumption that $S$ is CohenMacaulay and require only that $S$ has an isolated non-Cohen-Macaulay point. Then formula (17) holds for $n \gg 0$.

In order to prove Kawamata's Conjecture then, we must understand when core and graded core are equal. For an m-primary ideal generated by elements of the same degree in a Cohen-Macaulay graded ring over an infinite field, that core and graded core are equal follows from [6. Theorem 4.5]. But in general, this appears to be a subtle question. From the point of view of solving Kawamata's Conjecture (and understanding non-emptiness of linear systems more generally), this question is of great interest for ideals of the form $I=S_{\geq n}$ in a section ring. We return to this in a subsequent paper.

\section{REFERENCES}

[1] F. Ambro, Ladders on Fano varieties, Algebraic geometry, 9. J. Math. Sci. (New York) 94 (1999), no. 1, 1126-1135.

[2] I.M. Aberbach and C. Huneke, A theorem of Briançon-Skoda type for regular local rings containing a field, Proc. Amer. Math. Soc. 124 (1996), 707-713.

[3] Boutot, Jean-François Singularités rationnelles et quotients par les groupes réductifs, Invent. Math. 88 (1987), no. 1, 65-68.

[4] Bruns and Herzog, Cohen-Macaulay rings, Cambridge studies in advanced mathematics, 39, Cambridge UK, 1993. University Press,

[5] B. Conrad, Grothendieck Duality and Base Change Lecture Notes in Mathematics, 1750, Springer-Verlag, New York, 2000.

[6] A. Corso, C. Polini, and B. Ulrich, The structure of the core of ideals, Math. Ann. 321 (2001), no. $1,89-105$.

[7] Core and residual intersections of ideals, Trans. Amer. Math. Soc. 354 (2002) 25792594.

[8] J.P. Demailly, Multiplier ideal sheaves and analytic methods in algebraic geometry, School on Vanishing Theorems and Effective Results in Algebraic Geometry, Abdus Salam International Centre for Theoretical Physics, Trieste. (2000).

[9] L. Ein, Multiplier ideals, vanishing theorems and applications, Algebraic geometry-Santa Cruz 1995, 203-219, Proc. Sympos. Pure Math., 62, Part 1, Amer. Math. Soc., Providence, RI, 1997.

[10] L. Ein, R. Lazarsfeld, and K. Smith Uniform bounds and symbolic powers on smooth varieties ideals, Inventiones Math. 144 (2001), no. 2, 241-252.

[11] _Uniform behavior of valuation ideals preprint, 2002.

[12] H. Flenner, Rationale quasihomogene Singulatäten, Arch. Math. 36, 35-44 (1981).

[13] S. Goto and K. Nishida, The Cohen-Macaulay and Gorenstein Rees algebras associated to filtrations, Mem. Amer. Math. Soc. 526 (1994).

[14] S. Goto and K. I. Watanabe, On graded rings I, J. Math. Soc. Japan 30 (1978), no. 2, 179-213.

[15] S. Goto and Yamagishi, The theory of unconditioned strong d-sequences and modules of finite local cohomology, preprint

[16] A. Grothendieck and J. Dieudonné, Eléments de géométrie algébrique II, Publ. Math. I.H.E.S. 8 (1961).

[17] R. Hartshorne, Residues and duality, Springer Lecture Notes, vol. 20, Springer-Verlag, BerlinHeidelberg-New York, 1966.

[18] _ Algebraic Geometry, Springer Graduate Texts Springer-Verlag, Berlin-HeidelbergNew York, 1977.

[19] M. Herrmann, S. Ikeda, and U. Orbanz, Equimultiplicity and blowing up, Springer-Verlag, Berlin-Heidelberg-New York, 1988.

[20] M. Hochster and C. Huneke, Indecomposable canonical modules and connectedness, in Commutative algebra: syzygies, multiplicities, and birational algebra (South Hadley, MA, 1992), 197-208, Contemp. Math., 159, Amer. Math. Soc., Providence, RI, 1994. 
[21] J. Howald, Multiplier ideals of monomial ideals, Trans. Amer. Math. Soc. 353 (2001), no. 7, $2665-2671$.

[22] C. Huneke and I.Swanson, Cores of ideals in 2-dimensional regular local rings, Michigan Math. J. 42 (1995), 193-208.

[23] E. Hyry, Blow-up algebras and rational singularities, Manus. Math. 98 (1999), 377-390.

[24] Coefficient ideals and the Cohen-Macaulay property of Rees algebras, Proc. Amer. Math. Soc. 129 (2001), 1299-1308.

[25] Y. Kawamata, On effective non-vanishing and base-point-freeness, Asian Journal of Math, 4 (2000) 173-181.

[26] Y. Kawamata, Semipositivity, vanishing and applications, School on Vanishing Theorems and Effective Results in Algebraic Geometry, Abdus Salam International Centre for Theoretical Physics, Trieste. (2000).

[27] Y. Kawamata, K. Matsuda, and K. Matsuki, Introduction to the Minimal Model Program, Advanced Studies in Pure Mathematics, 10, Algebraic Geometry, Sendai 1985, (1987). pp 283-360.

[28] G. Kempf, F. Knudsen, D. Mumford, B. Saint-Donat, Toroidal embeddings, Lecture Notes in Mathematics, 339, Springer-Verlag, Berlin-New York, 1973.

[29] J. Kollár, Singularities of Pairs, Algebraic geometry-Santa Cruz 1995, 203-219, Proc. Sympos. Pure Math., 62, Part 1, Amer. Math. Soc., Providence, RI, 1997.

[30] J. Kollár, and S. Mori, Birational Geometry of Algebraic Varieties Cambridge University Press, 1999

[31] R. Lazarsfeld, Positivity in Algebraic Geometry, book in progress

[32] J. Lipman, Rational singularities, with applications to algebraic surfaces and unique factorization, Inst. Hautes tudes Sci. Publ. Math. No. 36 (1969) 195-279.

[33] Desingularization of two-dimensional schemes, Ann. of Math. 107 (1978), 151-207.

[34] - Cohen-Macaulayness in graded algebras, Math. Res. Letters 1 (1994), 149-157.

[35] _ Adjoints of ideals in regular local rings, Math. Res. Letters 1 (1994), 739-755.

[36] J. Lipman and B. Teissier, Pseudo-rational local rings and a theorem of Briançon-Skoda, Michigan Math. J. 28 (1981), 97-116.

[37] D. Rees and J. Sally, General elements and joint reductions, Michigan Math. J. 35 (1988), $241-254$.

[38] J. B. Sancho de Salas, Blowing-up morphisms with Cohen-Macaulay associated graded rings. Gomtrie algbrique et applications, I (La Rbida, 1984), 201-209, Travaux en Cours, 22, Hermann, Paris, 1987.

[39] P. Schenzel, Dualisierende Komplexe in der lokalen Algebra und Buchsbaum-Ringe, Lecture Notes in Mathematics, vol. 907, Springer-Verlag, Berlin-New York, 1982.

[40] V. V. Shokurov, A nonvanishing theorem. (Russian) Izv. Akad. Nauk SSSR Ser. Mat. 49 (1985), no. 3, 635-651.

[41] K. E. Smith, Fujita's freeness conjecture in terms of local cohomology, J. Algebraic Geom. 6 (1997), no. 3, 417-429.

[42] Y. T. Siu, Invariance of plurigenera, Invent. Math. 134 (1998), no. 3, 661-673.

[43] N. V. Trung, Toward a theory of generalized Cohen-Macaulay modules, Nagoya Math. J. 102 (1986), 1-49.

[44] 215 (1999), no. 2, 481-499.

[45] W.V. Vasconcelos, Aritmetic of blowup algebras, LMS Lect. Note Ser., vol. 195, Cambridge University Press, Cambridge, 1994.

[46] O. Zariski and P. Samuel, Commutative algebra, vol II, Springer Graduate Texts in Mathematics, vol. 29, Springer-Verlag, Berlin-Heidelberg-New York, 1975.

Department of Mathematics, PL 4 (Yliopistonkatu 5), Fin-00014 HELSingIN YLiOPisto, FinLAND

E-mail address: Eero.Hyry@helsinki.fi

Department of Mathematics, University of Michigan, Ann Arbor, Mi, 48109-1109

E-mail address: kesmith@umich.edu 